\title{
A survey for dwarf galaxy remnants around 14 globular clusters in the outer halo
}

\author{
A. Sollima, ${ }^{1 \star}$ D. Martínez Delgado, ${ }^{2}$ R. R. Muñoz, ${ }^{3}$ J. A. Carballo-Bello, ${ }^{4,5}$ \\ D. Valls-Gabaud, ${ }^{6}$ E. K. Grebel, ${ }^{2}$ F. A. Santana, ${ }^{3}$ P. Côté ${ }^{7}$ and S. G. Djorgovski ${ }^{8}$ \\ ${ }^{1}$ INAF Osservatorio Astronomico di Bologna, via Ranzani 1, I-40127 Bologna, Italy \\ ${ }^{2}$ Astronomisches Rechen-Institut, Zentrum für Astronomie der Universität Heidelberg, Mönchhofstr. 12-14, D-69120 Heidelberg, Germany \\ ${ }^{3}$ Departamento de Astronomía, Universidad de Chile, Camino El Observatorio 1515, Las Condes, 7591245 Santiago, Chile \\ ${ }^{4}$ Instituto de Astrofísica, Facultad de Física, Pontificia Universidad Católica de Chile, Av. Vicuña Mackenna 4860, Macul, 8970117 Santiago, Chile \\ ${ }^{5}$ Millenium Institute of Astrophysics, Camino El Observatorio 1515, Las Condes, 7591245 Santiago, Chile. Santiago, Chile \\ ${ }^{6}$ LERMA, CNRS UMR 8112, PSL, Observatoire de Paris, 61 Avenue de l'Observatoire, F-75014 Paris, France \\ ${ }^{7}$ National Research Council of Canada, Herzberg Astronomy and Astrophysics Program, 5071 W. Saanich Road, Victoria V9E 2E7, BC, Canada \\ ${ }^{8}$ Astronomy Department, California Institute of Technology, 1200 E. California Blvd, Pasadena, CA 91125, USA
}

Accepted 2018 February 23. Received 2018 February 2; in original form 2017 December 4

\begin{abstract}
We report the results of a systematic photometric survey of the peripheral regions of a sample of 14 globular clusters in the outer halo of the Milky Way at distances $d_{\mathrm{GC}}>25 \mathrm{kpc}$ from the Galactic Centre. The survey is aimed at searching for the remnants of the host satellite galaxies where these clusters could originally have been formed before being accreted on to the Galactic halo. The limiting surface brightness varies within our sample, but reaches $\mu_{\mathrm{V}, \lim }=30$ $32 \mathrm{mag} \operatorname{arcsec}^{-2}$. For only two globular clusters (NGC 7492 and Whiting 1; already suggested to be associated with the Sagittarius galaxy), we detect extended stellar populations that cannot be associated with either the clusters themselves or with the surrounding Galactic field population. We show that the lack of substructures around globular clusters at these Galactocentric distances is still compatible with the predictions of cosmological simulations whereby in the outer halo the Galactic globular cluster system is built up through hierarchical accretion at early epochs.
\end{abstract}

Key words: methods: observational-techniques: photometric-stars: Population IIglobular clusters: general-Galaxy: halo-Galaxy: structure.

\section{INTRODUCTION}

According to the most widely accepted cosmological model, the concordance $\Lambda$-cold dark matter $(\Lambda \mathrm{CDM})$ model (Planck Collaboration 2016) galaxies like the Milky Way are mainly formed by the hierarchical assembly of smaller subsystems (Pillepich, Madau \& Mayer 2015; Rodriguez-Gomez et al. 2016, and references therein).

A typical late accretion event would involve a dwarf satellite that is progressively disrupted by the tidal pull exerted by the parent galaxy. The stripped material (gas/stars/clusters/dark matter) is placed on orbits similar to that of the original satellite, hence forming tidal tails that may form multiple filamentary wraps around the parent galaxy (see Law, Johnston \& Majewski 2005). Accretion events in the inner halo at high redshift completed tens of orbits distributing their remnants homogeneously in space, while satellites accreted more recently and at large Galactocentric distances are expected to leave compact remnants. Gas-rich mergers constitute also the reservoir from which a homogeneous component of the halo could form in situ in the densest central region of the Milky Way. Models of galaxy formation predict that the haloes of Milky Way-like galaxies should therefore be characterized by a superposition of stellar populations from these substructures with a degree of discreteness that increases towards large Galactocentric distances (Bullock \& Johnston 2005; Cooper et al. 2010).

The tidal features left by the hierarchical formation process should be still observable in the outer halo and their detection provides an observational test of the $\Lambda \mathrm{CDM}$ paradigm. For this reason, in recent years huge observational efforts have been carried out to search for overdensities in large-scale photometric surveys like the Sloan Digital Sky Survey (SDSS; Abazajian et al. 2009), the Two-Micron All Sky Survey (2MASS; Skrutskie et al. 2006), and Pan-STARRS (Chambers et al. 2016). Truly spectacular examples have been observed both within the Milky Way, i.e. the case of the Sagittarius dwarf galaxy and its giant tidal stream (Ibata, Gilmore \& Irwin 1994; Majewski et al. 2003) and the 'field of streams' 
(Belokurov et al. 2006), in M31 (Ibata et al. 2001; McConnachie et al. 2009) and also in nearby galaxies (see Martínez-Delgado et al. 2010; Duc et al. 2015). Studies devoted to the quantification of the amount of substructures in the halo have been performed by Bell et al. (2008), Starkenburg et al. (2009), Xue et al. (2011), and Janesh et al. (2016), who indeed find that the halo is highly structured with an increasing clumpiness at large Galactocentric distances.

The outskirts of globular clusters (GCs) are among the best places to look for remnants of the ancient satellites predicted by the $\Lambda \mathrm{CDM}$ theory. Indeed, in the classical Searle \& Zinn (1978) scenario of the formation of the Galaxy, at least part of the outer halo GCs (at Galactocentric distances $d_{\mathrm{GC}}>8 \mathrm{kpc}$ ) formed in dwarf galaxies that were later accreted by the Milky Way. This hypothesis is based on the absence in the group of GCs populating the outermost Galactic halo of the clear metal abundance gradient observed in the inner Galaxy. An updated view of this evidence comes from the distribution of GCs in the age-metallicity plane (Marín-Franch et al. 2009; Forbes \& Bridges 2010; Leaman, VandenBerg \& Mendel 2013): GCs located at distances $d_{\mathrm{GC}}>15 \mathrm{kpc}$ tend to populate a separate branch of this diagram, away from the locus of nearby GCs. Moreover, these clusters exhibit peculiar kinematical properties (large, energetic orbits of high eccentricity), larger core radii, a higher specific frequency of RR Lyræ stars (Mackey \& Gilmore 2004), and most of them are distributed along an inclined disc that encompasses the Galaxy, together with the dwarf spheroidals and the ultra-faint dwarfs (Keller, Mackey \& Da Costa 2012; Pawlowski, Pflamm-Altenburg \& Kroupa 2012). The extra-Galactic origin of these clusters is also invoked to explain the bimodal metallicity distribution of GCs observed in both spiral and elliptical galaxies (Côté, Marzke \& West 1998; Côté et al. 2000; Kravtsov \& Gnedin 2005; Renaud, Agertz \& Gieles 2017). However, these trends do not provide a clear-cut proof of the origin of these GCs. On the other hand, if these clusters did form within larger stellar systems, they are expected to be surrounded by stellar populations of their host galaxy, over a wide field. Remarkably, recent studies have revealed the presence of streams in the surroundings of a few GCs in the inner halo (Martínez-Delgado et al. 2002; Bellazzini et al. 2003; Carballo-Bello et al. 2018a). In particular, the Sagittarius galaxy, the most prominent accretion event in the Milky Way, is expected to contribute to the Galactic GC system with $5 \div 9$ clusters, lying today along its orbital path (Law \& Majewski 2010a). Among them, the presence of the Sagittarius stellar population has been found in the surroundings of NGC 7492 and Whiting 1 (Carballo-Bello et al. 2014), at Galactocentric distances $d_{\mathrm{GC}}>25 \mathrm{kpc}$. The same scenario seems to be confirmed in M31 where all GCs beyond $30 \mathrm{kpc}$ from the centre appear to be associated with streams (Mackey et al. 2010; Veljanoski et al. 2014).

In this paper, we use deep wide-field photometric observations of a sample of 14 Galactic GCs populating the distant halo of the Milky Way (at distances $d_{G C}>25 \mathrm{kpc}$ ) to investigate the possible presence of stellar populations arising from their hypothetical progenitor hosts. In Section 2, we describe the observational material and the adopted data reduction techniques. In Section 3, we describe the methodology used to detect overdensities and the results of its application to our sample. Section 4 is devoted to the comparison of our results with a set of cosmological simulations. We discuss our results in Section 5.

\section{OBSERVATIONAL MATERIAL}

The GCs of our data base were selected among those populating the outer halo of the Milky Way at Galactocentric distances
$d_{G C}>25 \mathrm{kpc}$. Among the $19 \mathrm{GCs}$ listed in the Harris catalogue (Harris 1996, 2010 edition) in this distance range, we excluded two GCs at low Galactic latitude $|b|<10^{\circ}$ (Pyxis and Pal 2) because of the severe effect of differential reddening affecting their colourmagnitude diagrams (CMDs), two GCs (NGC 5824 and Pal 15) because their tidal extent exceeds the field of view of our data (see also Myeong et al. 2017; Kuzma, Da Costa \& Mackey 2018), and one GC (Ko 1) because of its sparse number of stars, which did not allow us to perform our analysis. The final sample hence consists of 14 GCs located in both hemispheres covering a range of distances of $25<d_{G C} / \mathrm{kpc}<125$ from the Galactic Centre.

The photometric data set consists of a set of images collected with the imagers Megacam [mounted at the CFHT (Canada-France Hawaii Telescope in Mauna Kea, Hawaii)] and Megacam (mounted at the Magellan II-Clay Telescope in Las Campanas, Chile) as a part of a project aimed at the characterization of Galactic GCs (Muñoz et al. 2018). This survey presents unprecedented photometric data quality for describing an almost complete sample of outer halo satellites, which was already used in many published works (see e.g. Bradford et al. 2011; Sollima et al. 2011a,b; Santana et al. 2013; Carballo-Bello et al. 2015). The camera Megacam at CFHT is a prime focus mosaic camera consisting of thirty-six $2048 \times 4612$ pixel CCDs (a total of 340 megapixels), covering a $0.96 \times 0.94$ square degree field of view with a pixel-scale of 0 ". 187 per pixels (Boulade et al. 2003). Observations were performed in queue mode during semesters 2009-A, 2009-B, and 2010-A mostly in photometric conditions. The average seeing was $\sim 0 " .8$. Clusters in the Southern hemisphere were observed using the Megacam camera at Magellan, which is composed of 36 closely packed CCDs assembled in an $18432 \times 18432$ pixels array. The CCDs have $13.5 \mu \mathrm{m}$ square pixels that subtend 0 ".08 at the $f / 5$ focus, yielding a 25 arcmin $\times 25$ arcmin field of view. The average seeing of these observations was $\sim 0$ ". 9 . For each cluster, multiple images were observed through the Sloan $g^{\prime}$ and $r^{\prime}$ filters, placing the cluster close to the centre of the camera field of view. The log of the observations, including exposure times, number of exposures, and overall field of view for each target cluster is listed in Table 1. Standard photometric reduction (bias subtraction, flat-field, etc.) was carried out using the $\operatorname{IRAF}^{1}$ task CCDPROC.

The photometric analysis was carried out using the PSF (point spread function)-fitting algorithm of the DAOPHOT/ALLFRAME package (Stetson 1994). The brightest and most isolated stars were used to construct a model PSF and to link PSF and aperture magnitudes. Images were aligned and stacked to construct a high signal-to-noise image where the source detection was performed. The photometric analysis was then done on the individual images and the resulting magnitudes were averaged. For the objects in common with the SDSS-DR7 catalogue (Abazajian et al. 2009), the instrumental magnitudes were calibrated using the stars in common, selecting the ones with $18<r_{\text {sdss }}<21.5$ and $18<g_{\text {sdss }}<22$ to avoid both saturated and large photometric uncertainty stars. The objects that lay outside the SDSS footprint were calibrated using the average extinction terms, zero-points, and the colour-terms determined for the GCs overlapping the SDSS imaging area and that were observed with the same instrument under the same observing conditions. To limit the contamination from background galaxies, whose location in the CMD partly overlaps the locus of the MS of a halo stellar

\footnotetext{
${ }^{1}$ IRAF is distributed by the National Optical Astronomy Observatories, which are operated by the Association of Universities for Research in Astronomy, Inc., under cooperative agreement with the National Science Foundation.
} 
Table 1. Summary of observations.

\begin{tabular}{|c|c|c|c|c|c|}
\hline Name & Telescope & Filter & $N_{\exp }$ & $\begin{array}{l}t_{\exp } \\
(\mathrm{s})\end{array}$ & $\begin{array}{c}\text { FoV } \\
\left(\mathrm{deg}^{2}\right)\end{array}$ \\
\hline \multirow[t]{2}{*}{ Pal 13} & \multirow[t]{2}{*}{ CFHT } & $g$ & 6 & 360 & 0.98 \\
\hline & & $r$ & 6 & 360 & 0.98 \\
\hline \multirow[t]{4}{*}{ NGC 7492} & \multirow[t]{2}{*}{ CFHT } & $g$ & 6 & 120 & 0.94 \\
\hline & & $r$ & 6 & 120 & 0.94 \\
\hline & \multirow[t]{2}{*}{ Clay } & $g$ & 5 & 90 & 0.21 \\
\hline & & $r$ & 5 & 180 & 0.21 \\
\hline \multirow[t]{2}{*}{ Whiting 1} & \multirow[t]{2}{*}{ CFHT } & $g$ & 6 & 300 & 0.95 \\
\hline & & $r$ & 6 & 300 & 0.95 \\
\hline \multirow[t]{2}{*}{ NGC 6229} & \multirow[t]{2}{*}{ CFHT } & $g$ & 6 & 90 & 0.91 \\
\hline & & $r$ & 6 & 90 & 0.91 \\
\hline \multirow[t]{2}{*}{$\mathrm{AM} 4$} & \multirow[t]{2}{*}{ Clay } & $g$ & 5 & 90 & 0.21 \\
\hline & & $r$ & 5 & 180 & 0.21 \\
\hline \multirow[t]{2}{*}{ Koposov 2} & \multirow[t]{2}{*}{ CFHT } & $g$ & 6 & 500 & 0.94 \\
\hline & & $r$ & 6 & 500 & 0.94 \\
\hline \multirow[t]{2}{*}{ NGC 5694} & \multirow[t]{2}{*}{ CFHT } & $g$ & 6 & 60 & 0.95 \\
\hline & & $r$ & 6 & 60 & 0.95 \\
\hline \multirow[t]{2}{*}{ NGC 7006} & \multirow[t]{2}{*}{ CFHT } & $g$ & 6 & 240 & 0.95 \\
\hline & & $r$ & 6 & 240 & 0.95 \\
\hline \multirow[t]{2}{*}{ Pal 14} & \multirow[t]{2}{*}{ CFHT } & $g$ & 6 & 680 & 0.95 \\
\hline & & $r$ & 9 & 680 & 0.95 \\
\hline \multirow[t]{2}{*}{ NGC 2419} & \multirow[t]{2}{*}{ CFHT } & $g$ & 6 & 450 & 1.02 \\
\hline & & $r$ & 6 & 450 & 1.02 \\
\hline \multirow[t]{2}{*}{ Eridanus } & \multirow[t]{2}{*}{ CFHT } & $g$ & 6 & 270 & 0.94 \\
\hline & & $r$ & 6 & 270 & 0.94 \\
\hline \multirow[t]{4}{*}{ Pal 3} & \multirow[t]{2}{*}{ CFHT } & $g$ & 6 & 270 & 0.98 \\
\hline & & $r$ & 6 & 270 & 0.98 \\
\hline & \multirow[t]{2}{*}{ Clay } & $g$ & 5 & 90 & 0.21 \\
\hline & & $r$ & 5 & 180 & 0.21 \\
\hline \multirow[t]{2}{*}{$\mathrm{Pal} 4$} & \multirow[t]{2}{*}{ CFHT } & $g$ & 6 & 440 & 0.96 \\
\hline & & $r$ & 6 & 440 & 0.96 \\
\hline \multirow[t]{2}{*}{ AM 1} & \multirow[t]{2}{*}{ Clay } & $g$ & 5 & 90 & 0.21 \\
\hline & & $r$ & 5 & 180 & 0.21 \\
\hline
\end{tabular}

population, we removed all stars with a sharpness parameter $|S|>0.3$. This value was chosen to include the bulk of bona-fide cluster MS stars selected within $\Delta(g-r)<0.1$ about the MS ridge line and located within $R<r_{\text {lim }}$. This criterion has been found to provide the best compromise between completeness and contamination with a better discrimination power than other considered alternatives (e.g. PSF-fitting versus aperture magnitude, etc.).

The $(g-r)-g$ CMDs of the 14 GCs of our sample are shown in Fig. 1. They sample the cluster population from the tip of the red giant branch to some 1-4 mag below the main-sequence (MS) turnoff depending on the cluster distance, reaching a typical magnitude limit of $g \sim 25.5$. At large distances from the centres of the clusters the stellar populations of the Galactic field dominate, showing a fairly homogeneous distribution of stars in the blue portion of the CMD (at $g-r<1$; composed of the superposition of disc/halo upper MS stars at different heliocentric distances) and a red plume (at $g-r>1$; composed of low-mass MS dwarfs). In a few clusters (e.g. NGC 7492, Whiting 1), an overdensity of stars in the blue region of the CMD resembling a MS feature is apparent. At faint magnitudes (at $g>24$ and $g-r<0.6$ ), a large number of background galaxies contaminate the CMD. Artificial star experiments were performed on the science images of NGC 2419 and NGC 7006 indicating a completeness level $>80$ percent at $g \sim 24$ in both clusters, with a sharp decrease at fainter magnitudes.

\section{METHOD}

The CMDs shown in Fig. 1 were used to detect any possible overdensity of stars compatible with that produced by the stellar population of a surrounding remnant.

In the following analysis, magnitudes were dereddened using the reddening predicted by Schlafly \& Finkbeiner (2011) and the extinction coefficients by Schlegel, Finkbeiner \& Davis (1998).

To avoid the contamination from the stars of the GC itself, we only considered in our analysis stars located beyond a limiting radius $r_{\text {lim }}$, where the cluster contribution vanishes. For this purpose, we selected stars within three times the colour standard deviation about the MS ridge line and counted the number of selected objects in concentric annuli. The density profile was then derived by dividing the number counts by the area of each annulus. Number densities were then converted into magnitudes and scaled to match the surface brightness profile of Trager, King \& Djorgovski (1995). For those clusters not included in the Trager et al. (1995) sample, we adopted the best-fitting King (1966) models provided by Carballo-Bello et al. (2014, Whiting 1 and AM 4), Ibata et al. (2011, NGC 2419), and Harris (1996, Eridanus), which were normalized to match the integrated $V$ magnitude listed in the Harris (1996) catalogue. The resulting surface brightness profiles are shown in Fig. 2. In all clusters the profiles show the typical declining trend and flatten at a value beyond which the Galactic contribution dominates over the clusters' stellar population. For different choices of $r_{\text {lim }}$, we performed a $\chi^{2}$ test by selecting radial bins at projected distances $R>r_{\mathrm{lim}}$. We chose $r_{\text {lim }}$ as the minimum distance beyond which the associated $\chi^{2}$ is compatible with a constant density at the 99.8 per cent confidence level. The mean surface brightness calculated at distances $R>r_{\text {lim }}$ was then assumed to be an upper limit for the surface brightness of the hypothetical underlying remnant $\left(\mu_{\mathrm{V}, \max }\right)$. Indeed, this approximate limit depends on a number of assumptions. For example, a direct number count/surface brightness conversion was assumed, neglecting possible differences in the stellar content of the sampled population (e.g. age, metallicity, mass function, etc.). However, the cluster and its hypothetical host galaxy are expected to be characterized by similar (old and metal-poor) stellar populations at the same mean heliocentric distance (although with a larger spread for the remnant) and are affected by the same dust extinction, thus sharing the same location in the CMD. Note that while any stellar population with a surface brightness brighter than $\mu_{\mathrm{V} \text {, max }}$ would have produced a plateau at a surface brightness level larger than observed, the estimated background level could have been spuriously increased by (i) residual contamination from unresolved galaxies, (ii) Poisson fluctuations hampering the detection of any declining trend at low number counts, and (iii) the contribution of the remnants of other disrupted satellites lying along the line of sight to the GC. The adopted values of $r_{\text {lim }}$ and $\mu_{\mathrm{V} \text {, max }}$ for the analysed GCs are listed in Table 2. The derived background surface brightnesses lie in the range $29.4<\mu_{\mathrm{V} \text {, max }}<31.7 \mathrm{mag}$ $\operatorname{arcsec}^{-2}$ depending on the sampled area and the cluster distance, and have typical uncertainties of $\sim 0.5$ mag $\operatorname{arcsec}^{-2}$.

Contamination from background galaxies has been significantly reduced by the strict cut in the sharpness parameter described in Section 2. While this criterion is effective in removing extended objects at bright magnitudes, it cannot avoid some residual contamination at faint magnitudes $(g>24)$ where the uncertainties in the stellar profile determination produce an overlap between stars and galaxies also in the sharpness parameter domain. Moreover, because of such a strict criterion, many faint stars at $g>24$ are removed causing a drop of the photometric completeness. Because 

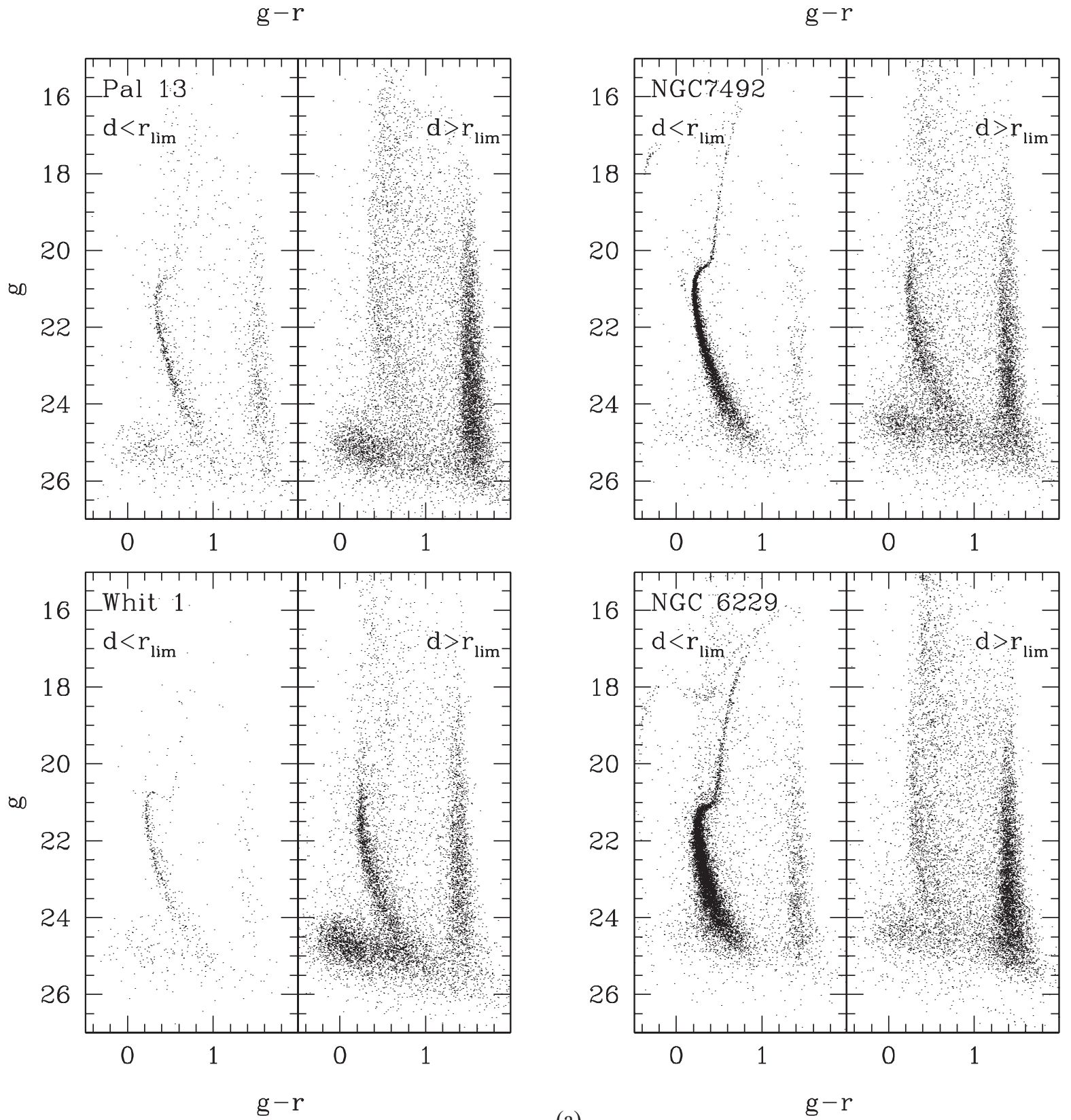

Figure 1. (a) Colour-magnitude diagrams in $(g, g-r)$ of Pal 13, NGC 7492, Whiting 1, and NGC 6229. The left-hand panels refer to the region within the estimated cluster-limiting radius $r_{\mathrm{lim}}$ (see Section 3 ). The right-hand panels refers to the region beyond $r_{\text {lim. }}$. Only stars within the adopted sharpness threshold are shown. (b) Colour-magnitude diagrams for AM 4, Ko 2, NGC 5694, and NGC 7006. (c) Colour-magnitude diagrams for Pal 14, NGC 2419, Eridanus, and Pal 3. (d) Colour-magnitude diagrams for Pal 4 and AM 1.

of this effect, a quantitative analysis was only carried out for the subsample of eight GCs within a heliocentric distance $d_{\odot}<45 \mathrm{kpc}$.

To quantitatively test for the presence of an overdensity of MS stars around the GCs of our sample, we adopted three different methods.

First, we compared the number of stars contained in a region of the CMD encompassing the predicted location of halo MS stars at the cluster distance with that predicted by the GALAXIA Galactic model (Sharma et al. 2011, method A). For this purpose, we counted the number of stars with colours within $3 \sigma$ about the cluster MS ridge line and within the magnitude interval $20<g_{0}<24$ in both the observed $\left(N_{\text {obs }}\right)$ and the synthetic $\left(N_{\text {mod }}\right)$ CMD. In this range the photometric completeness is $>80$ per cent and the contamination from unresolved galaxies is negligible. The statistical significance of any density difference was estimated as the number of standard deviations with respect to the zero difference hypothesis, assuming Poisson noise for number counts in the regime of large $N(\sigma(N) \sim$ $\sqrt{N}$ ) and no covariance between $N_{\text {obs }}$ and $N_{\text {mod }}$ :

$Q=\frac{A_{\text {mod }} N_{\text {obs }}-A_{\text {obs }} N_{\text {mod }}}{\sqrt{A_{\text {mod }}^{2} N_{\text {obs }}+A_{\text {obs }}^{2} N_{\text {mod }}}}$ Cerviño et al. (2002),

where $A_{\mathrm{obs}}$ and $A_{\text {mod }}$ are the sampled areas in the observed and synthetic samples, respectively. The values of $Q$ are listed in Table 2. A comparison between the observed CMD of NGC 6229 and the 
$g-r$
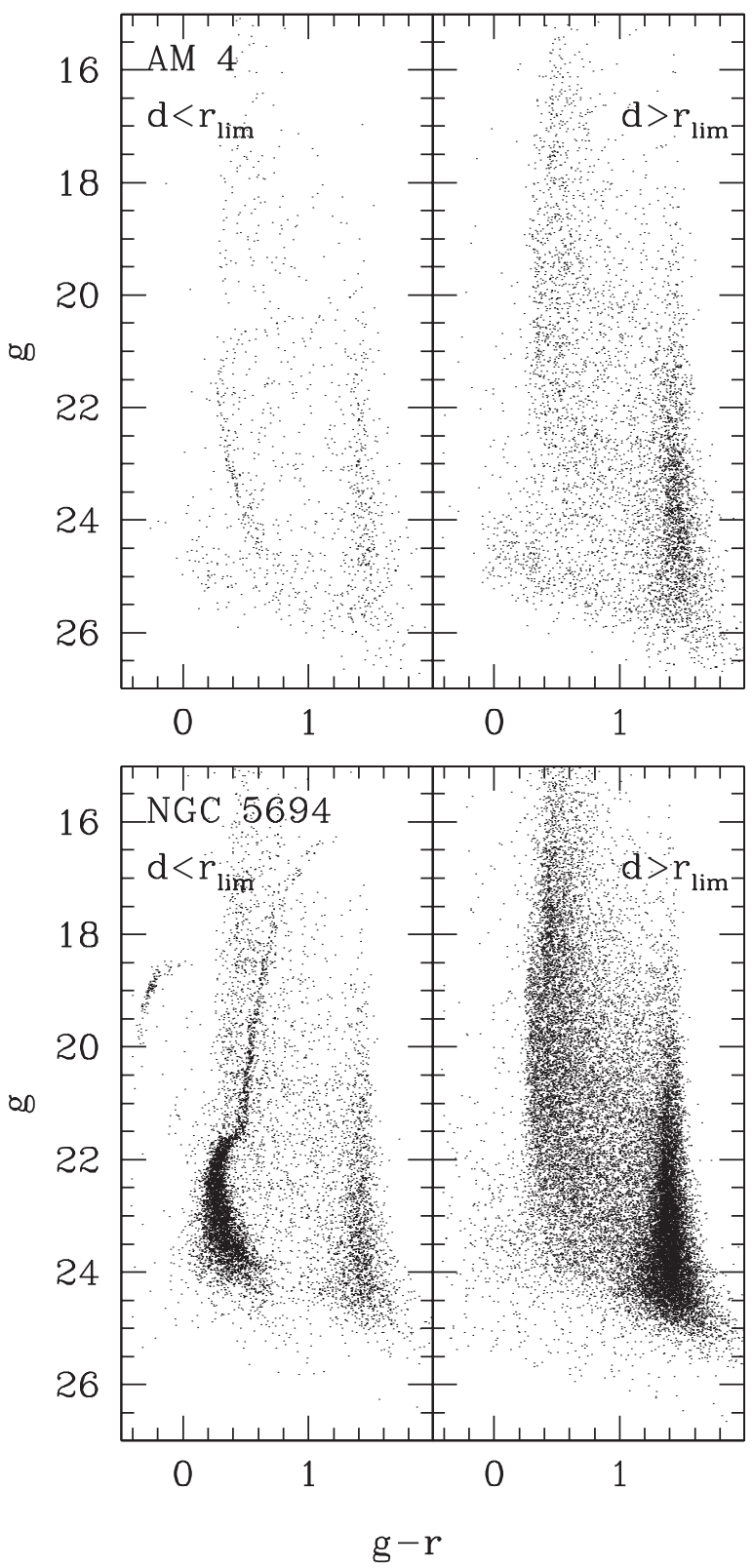

$g-r$
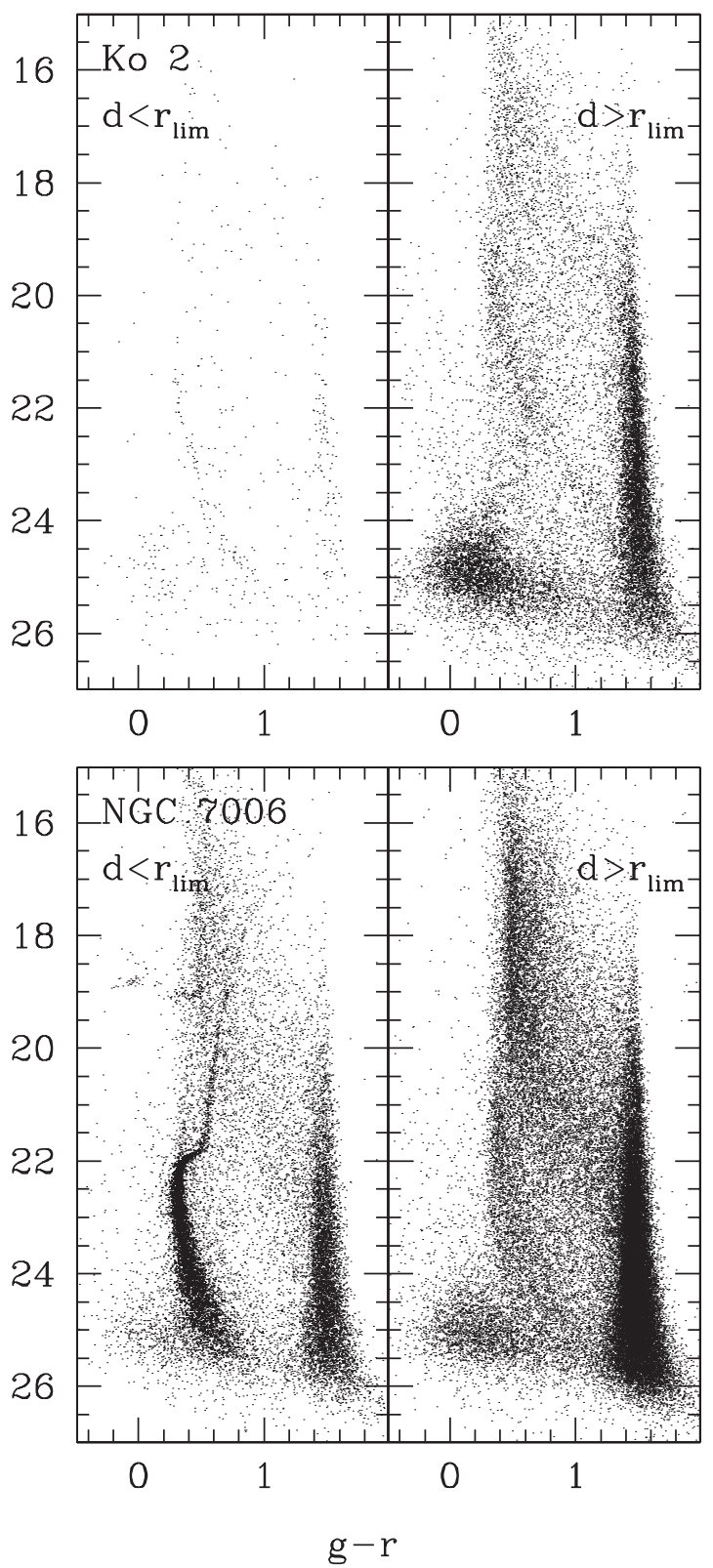

Figure 1 - continued

synthetic one predicted by the GALAXIA model in the same direction is shown in Fig. 3, as an example. While on average number counts are overestimated by the GALAXIA model, and yield negative values for $Q$, highly significant overdensities $(Q>3)$ are found around NGC 7492 and Whiting 1 (see Section 5).

These detections strongly depend on the adopted Galactic model. We hence also consider two alternative model-independent approaches. In the former approach (method B), we count the number of stars contained in three regions of the CMD defined as follows (see Fig. 4):

(i) A region defined by the intersection between a selection box in the intervals $0.1<(g-r)_{0}<0.5$ and $21<g_{0}<24$, and the portion of the CMD contained between the loci of a theoretical isochrone of the PARSEC-COLIBRI data base (Marigo et al. 2017) with $Z=0.0005$ and $t=10 \mathrm{Gyr}$ (hereafter referred as 'reference isochrone') where the absolute magnitudes were converted into the observational ones by adding the distance moduli corresponding to distances of 20 and $45 \mathrm{kpc}$. This region includes mainly halo MS stars in this interval of distances;

(ii) A region containing all stars in the intervals $0.1<(g-r)_{0}<0.5$ and $17.5<g_{0}<20.5$. This region includes mainly nearby thin and thick disc MS stars;

(iii) A region containing all stars in the intervals $1.2<(g-r)_{0}<1.6$ and $18<g_{0}<24$. This region includes low-mass MS stars of the Galactic disc(s).

Since these regions contain stars belonging to different Galactic components, the number count ratios are expected to be functions of the Galactic coordinates. In particular, in absence of any 
$g-r$
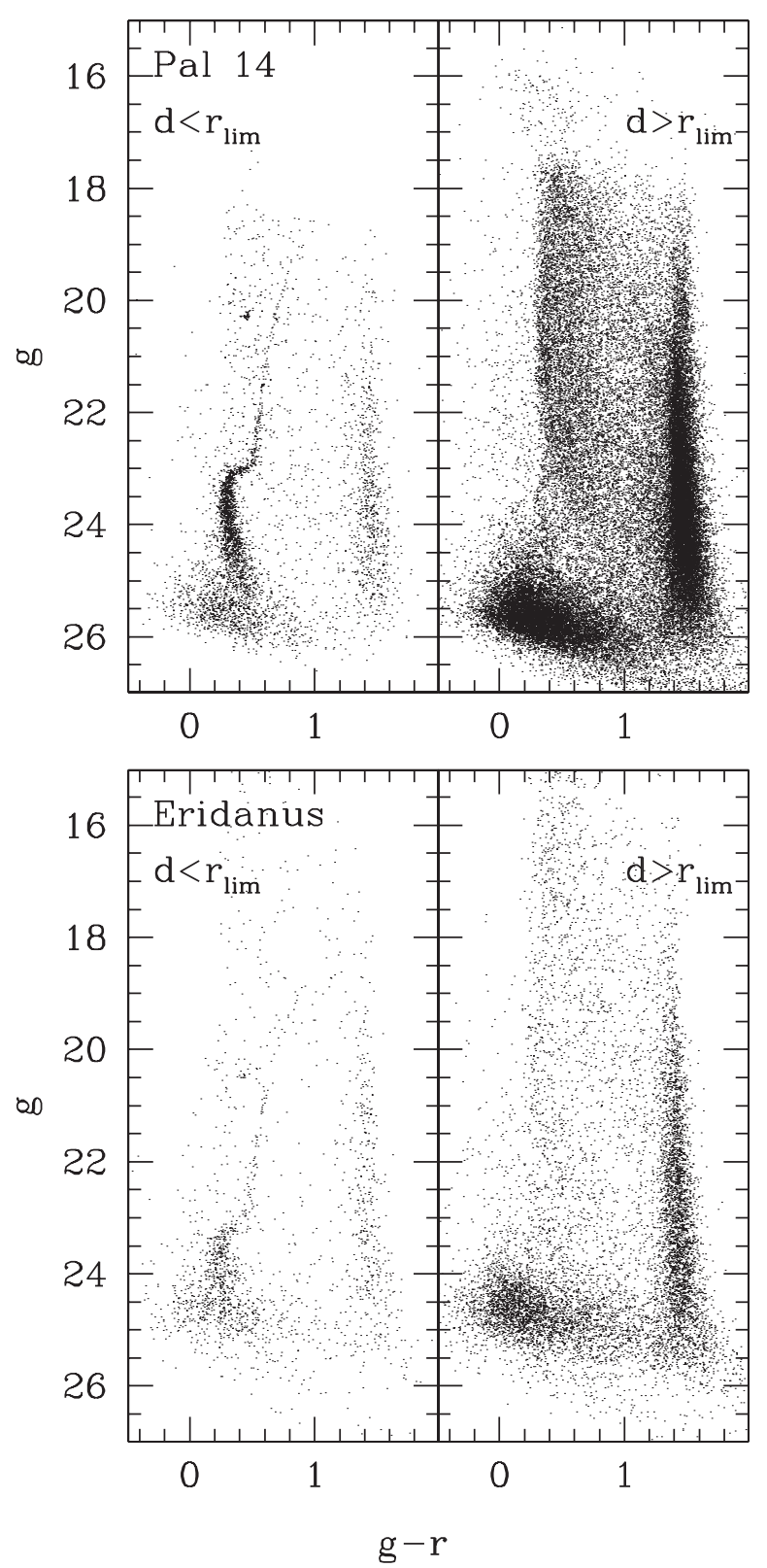

$g-r$
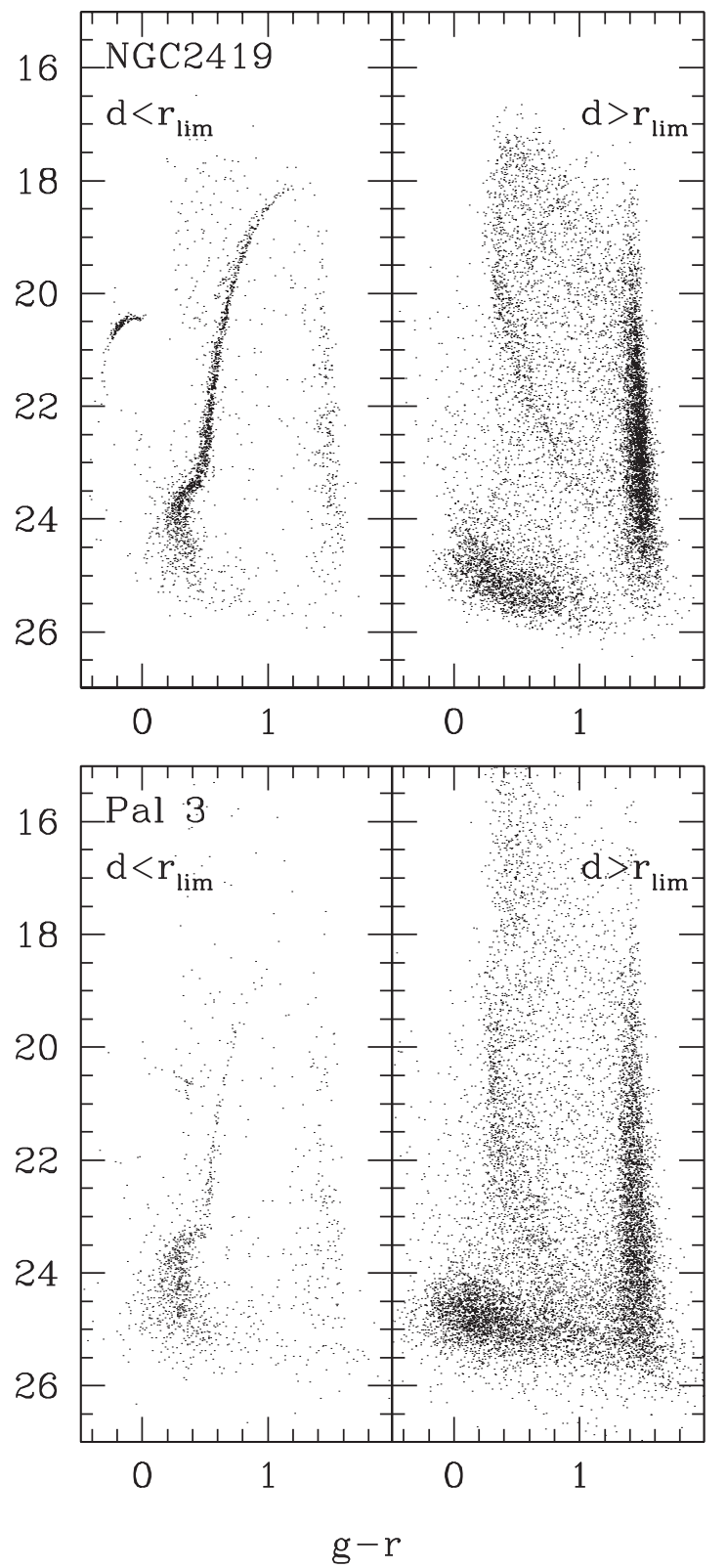

Figure 1 - continued

substructure, the larger the Galactic latitude the smaller the portion of disc stars along the line of sight. On the other hand, around clusters immersed in large overdensities, a significant increase above the mean trend is expected to be noticeable. The number ratios $N_{\mathrm{A}} / N_{\mathrm{B}}$ and $N_{\mathrm{A}} / N_{\mathrm{C}}$ for the GCs of our sample as a function of the Galactic latitude are shown in Fig. 4. The majority of GCs define smoothly increasing relations when both number count ratios are considered. Two clusters stray from these relations: NGC 7492 and Whiting 1, which both show an excess with respect to the global trend.

The third and last technique adopted to detect overdensities (method C) aims at studying the 3D density profile of halo MS stars along the line of sight of each GC. For this purpose, we selected stars in the colour range $0.1<(g-r)_{0}<0.5$ and estimated their corresponding absolute magnitude by interpolating along the reference isochrone. The distance modulus (DM) of each star was then calculated and a histogram was constructed $N(\mathrm{DM})$. The corresponding $3 \mathrm{D}$ density profile becomes

$\log \rho\left(d_{\odot}\right)=\log N(\mathrm{DM})-0.6 \mathrm{DM}$.

Here, we are neglecting the effect of photometric errors and of individual age/metallicity differences, which can spread the distribution up to $0.3 \mathrm{mag}$. For this reason, the above density profile can only be used to study large-scale trends. However, the presence of a compact stellar population surrounding the cluster would produce an increase of the local density at a distance similar to that of the cluster, which should be noticeable in the density profile. The derived 3D density profiles for the GCs of our sample are shown in Fig. 5. A power law was fitted to the derived density profiles excluding the interval surrounding the cluster (at $\Delta \log (d / \mathrm{kpc})<0.1)$. Again, while in the majority of the analysed GCs the density profile is well 


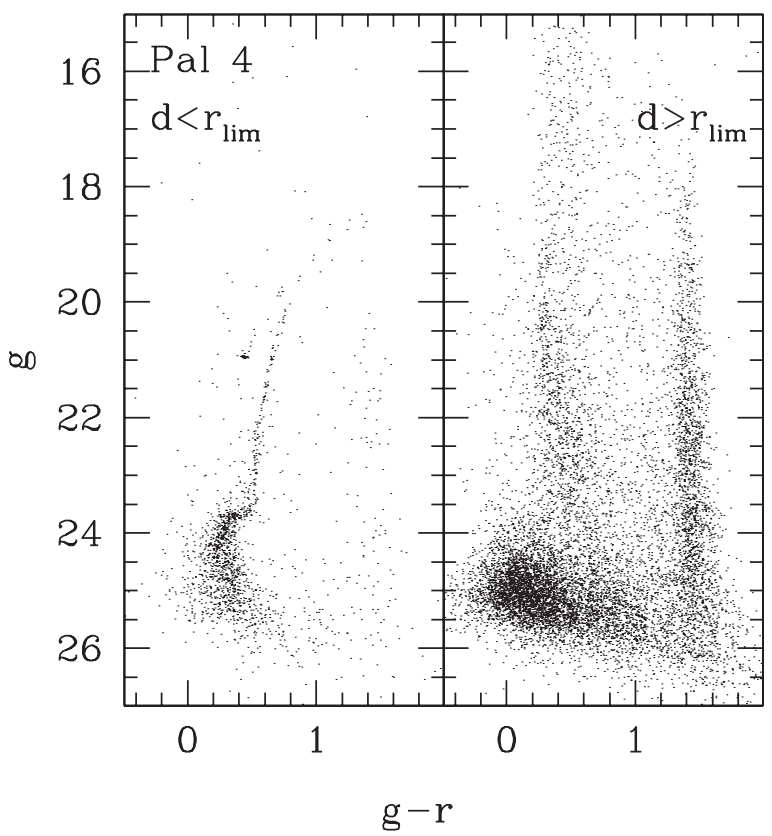

(d)

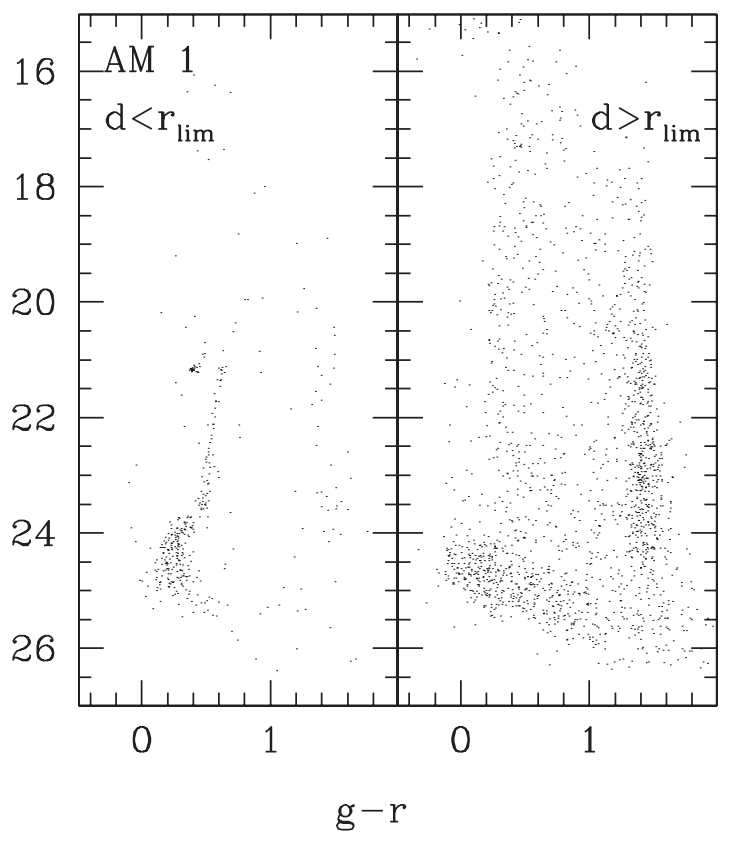

Figure 1 - continued

described by a single power law, a bump at distances compatible to those of the corresponding clusters is apparent around NGC 7492 and Whiting 1.

In Fig. 6, the 2D density map of NGC 7492 and Whiting 1 is shown. These maps are constructed by applying the $k$-nearest neighbour density estimator (Silverman 1986, with $k=10$ ) to the sample of stars with colours within $3 \sigma$ about the cluster MS ridge line. Note that, outside the adopted limiting radius, only barely significant overdensities homogeneously distributed across the field of view are apparent.

Summarizing, the three independent methods outlined in this section indicate the presence of significant overdensities of MS stars around NGC 7492 and Whiting 1, which are located in a small interval of distances compatible with those of these GCs. These stellar populations have a flat spatial distribution across the analysed field of view extending far beyond the tidal radii (see Figs 2 and 6) and present CMDs that are inconsistent with those predicted by Galactic models. We therefore conclude that the detected stellar populations are due to the remnants of dwarf galaxies within which these two GCs are embedded. In particular, these two GCs are known to be located along the path of the Sagittarius stream, and the presence of the stellar population of this remnant in the surroundings of these two GCs has been revealed by previous works (CarballoBello et al. 2014, 2017, 2018b).

\section{COMPARISON WITH COSMOLOGICAL SIMULATIONS}

In the previous section we presented evidence for significant overdensities around two (out of eight) GCs at $d_{\odot}<45 \mathrm{kpc}$, and provided upper limits for the surface brightness of any undetected underlying stellar populations around each of the $14 \mathrm{GCs}$ of the entire sample. At first glance, this evidence could appear in contrast with the $\Lambda \mathrm{CDM}$ paradigm predicting that most of the Milky Way halo stars and GCs at large Galactocentric distances were accreted from infalling satellites. Note, however, that the analysis performed in Section 3 is designed to detect overdensities above an average background. Such a background could itself consist of by the superposition of the remnants of many satellites accreted during a Hubble time (Deason, Belokurov \& Weisz 2015). So, the existence of GCs with negative detections does not constitute a violation of the $\Lambda \mathrm{CDM}$ model. In this context, it is interesting to compare this result with the predictions of $\Lambda \mathrm{CDM}$ cosmological simulations.

We used the set of publicly available catalogues of synthetic stars by Lowing et al. (2015), which are based on the post-processing of the Aquarius project simulation (Springel et al. 2008). These simulations assume the standard $\Lambda \mathrm{CDM}$ cosmology with parameters chosen to be consistent with the results from the WMAP 1-yr data (Spergel et al. 2003) and the 2dF Galaxy Redshift Survey data (Colless et al. 2001), namely matter density parameter $\Omega_{\mathrm{M}}=0.25$; cosmological constant $\Omega_{\Lambda}=0.75$; power spectrum normalization $\sigma_{8}=0.9$; spectral index $n_{\mathrm{s}}=1$; and Hubble parameter $h=0.73$. Five Milky Way-like haloes (Aquarius A-E) were selected randomly from a set of isolated $\sim 10^{12} \mathrm{M}_{\odot}$ haloes identified in a lower resolution parent simulation of a $100 \mathrm{~h}^{-1} \mathrm{Mpc}^{3}$ volume (Gao et al. 2008). The mass, age, and chemical composition of stars formed in each particle of the simulation was calculated using the Durham semi-analytic model GALFORM (Cole et al. 2000), which adopts physically motivated recipes to follow the chemical evolution of gas and stars including gas cooling and feedback effects. This model produces a satellite luminosity function that matches that of the Milky Way and also generates stellar populations in satellites that match the observed luminosity-metallicity relation. A particle-tagging algorithm was then adopted to associate the stellar component with six-dimensional phase-space volumes. As a final step, the total number of stars associated with each tagged particle was calculated and associated with a corresponding synthetic population (characterized by age, metallicity, and magnitudes) using the technique described by Pasetto, Chiosi \& Kawata (2012) and the stellar isochrones of the PARSEC data base (Bressan et al. 2012).

We tested the hypothesis that all the considered GCs were originally part of accreted satellites. For each GC of our sample, we randomly extracted a particle from one of the above simulations among those formed $>10 \mathrm{Gyr}$ ago, belonging to the main halo at 

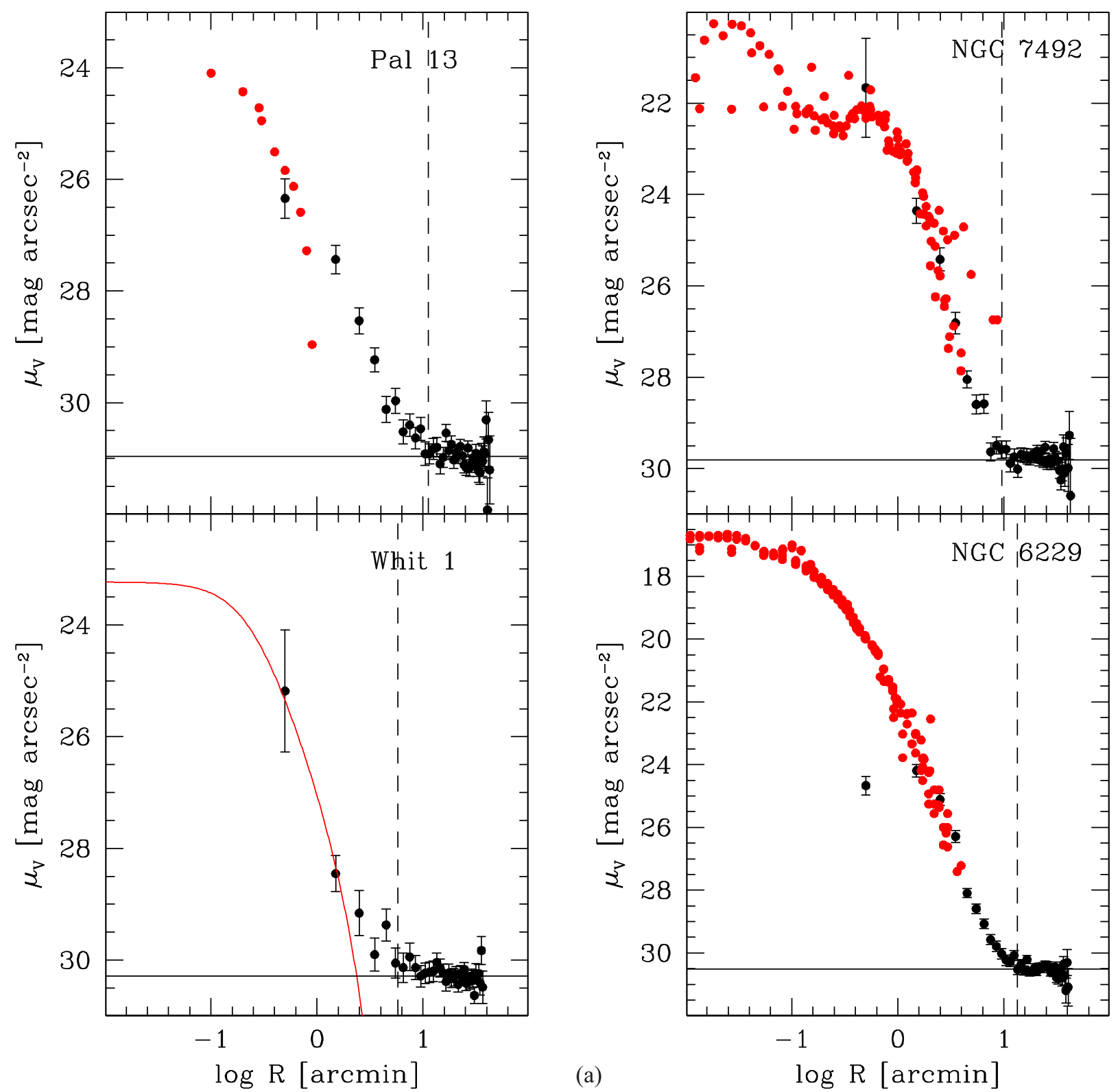

Figure 2. (a) Surface brightness profiles of Pal 13, NGC 7492, Whiting 1, and NGC 6229. Black points are measures from this work, red points (grey in the printed version of the paper) are from Trager et al. (1995). The solid red curve represents the best-fitting King (1966) model. The adopted limiting radius $r_{\text {lim }}$ and the background surface brightness $\mu_{\mathrm{V}}$, lim are marked by the vertical dashed line and the horizontal solid line, respectively. (b) Surface brightness profiles of AM 4, Ko 2, NGC 5694, NGC 7006, and Pal 14. (c) Surface brightness profiles of NGC 2419, Eridanus, Pal 3, Pal 4, and AM 1.

the present day and at a Galactocentric distance within 10 per cent of that of the considered GC, and marked it as a 'synthetic GC'. With this choice we neglect all the complex and uncertain processes in the context of the formation and survival of GCs in different environments (see e.g. Griffen et al. 2010), implicitly assuming that the GC formation efficiency is constant in the satellites that formed the Galactic halo. While this last assumption is clearly not suitable to study the general properties of GCs, it is not crucial for the purpose of quantifying the lumpiness of the halo. All the particles of the simulation were rotated in order to bring the synthetic GC at the same Galactic coordinates of the considered cluster, adopting a position for the Sun at $(X, Y, Z)=(8,0,0) \mathrm{kpc}$. The distance moduli $\left(\mathrm{DM}_{i}\right)$ of the particles located within a projected position on the sky of 1 square degree were calculated and added to their $g$ absolute magnitudes $\left(M_{\mathrm{g}, \text { syn }}\right)$, thus constructing a synthetic CMD

$g_{\text {syn }}=M_{\mathrm{g}, \text { syn }}+\mathrm{DM}_{i}$

The number $N_{\text {syn }}$ of stars contained within $3 \sigma$ about the cluster MS ridge line and in the magnitude range $3.92<g_{\text {syn }}-\mathrm{DM}_{\mathrm{GC}}<6$ was counted, where $\mathrm{DM}_{\mathrm{GC}}$ is the DMs of the considered GC. To express the projected density in units of surface brightness, the total $V$-band luminosity of the stellar population was calculated as:

$M_{\mathrm{V}, \text { tot }}=-2.5 \log \left(\sum_{i} 10^{-0.4 M_{\mathrm{V}, i}}\right)$. 

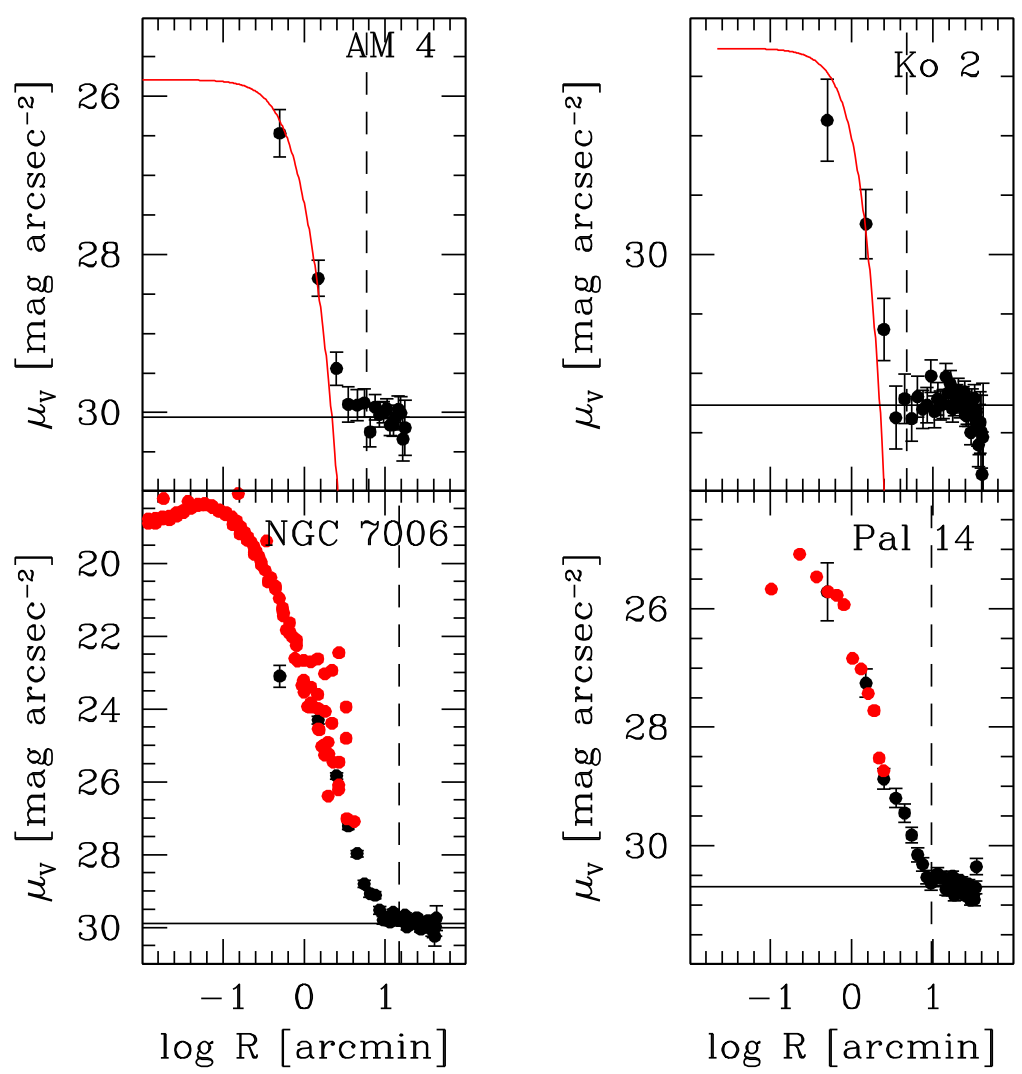

(b)

Figure 2 - continued

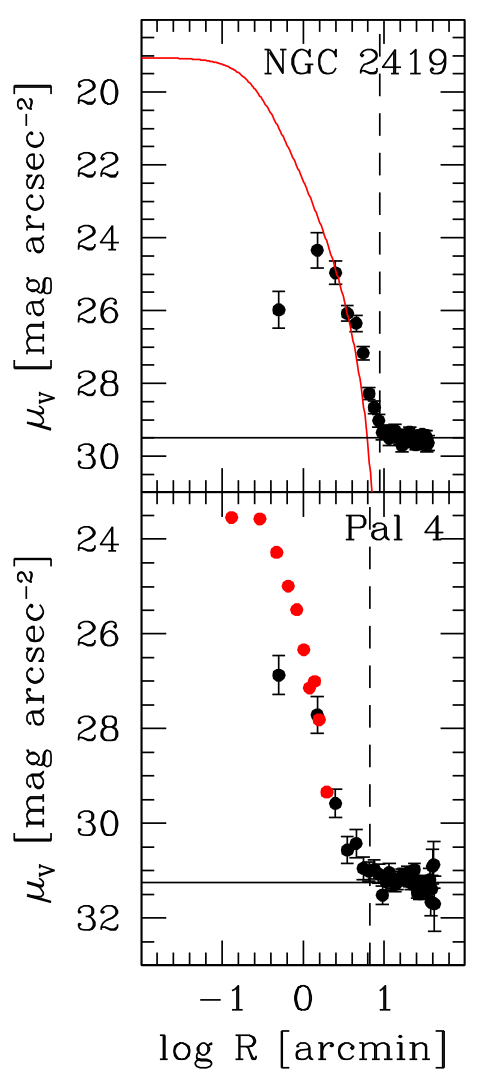

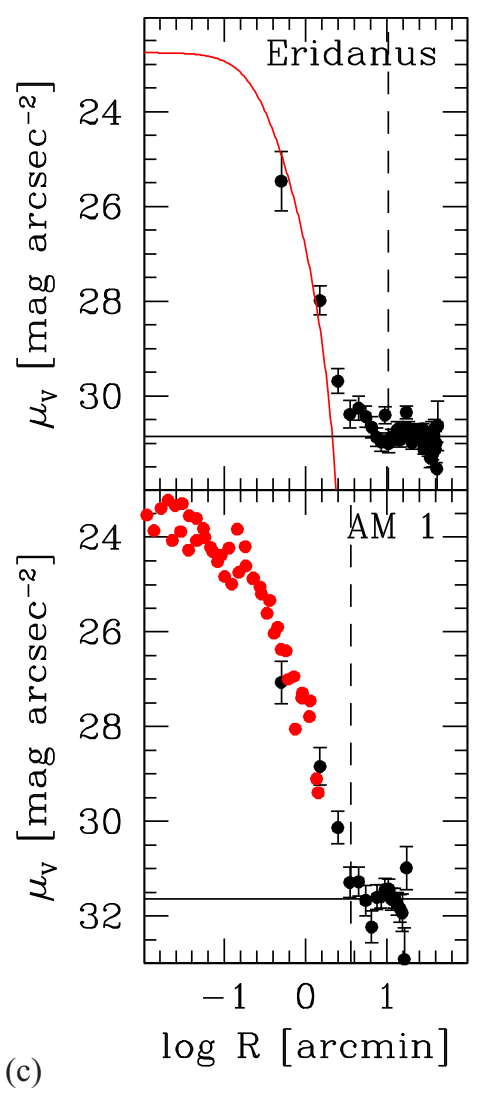

Figure 2 - continued
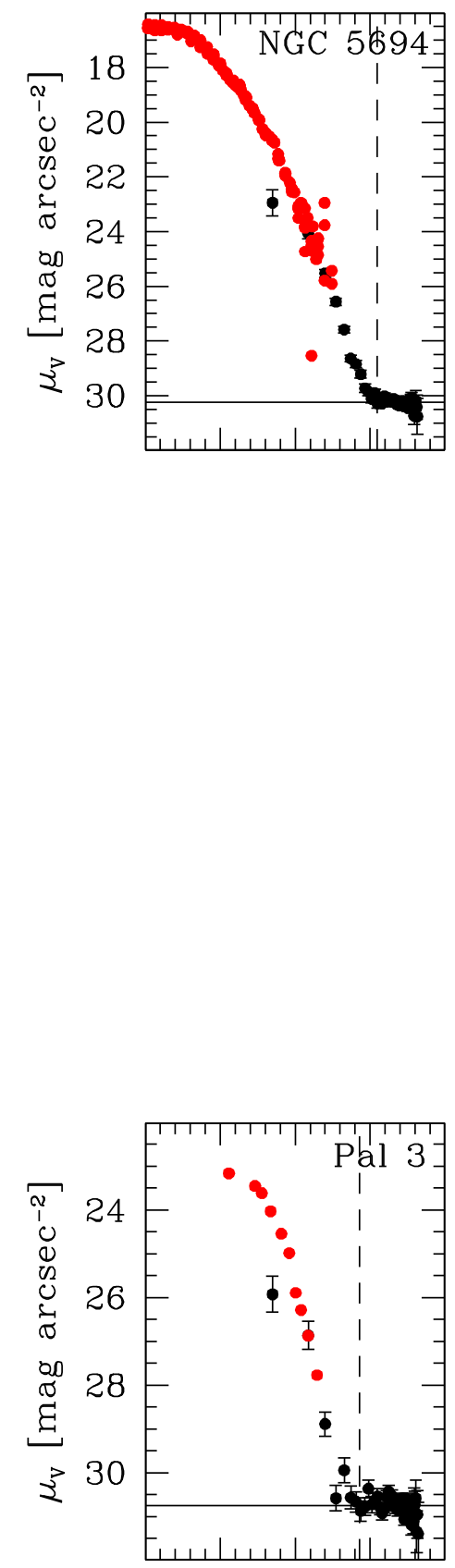
Table 2. Heliocentric and Galactocentric distance, Galactic latitude, estimated limiting radius, background surface brightness, and index $Q$ of the clusters of our sample.

\begin{tabular}{lcrrrcr}
\hline Name & $\begin{array}{c}d_{\odot} \\
(\mathrm{kpc})\end{array}$ & $\begin{array}{c}d_{\mathrm{GC}} \\
(\mathrm{kpc})\end{array}$ & \multicolumn{1}{c}{$\begin{array}{c}b \\
\left.{ }^{\circ}\right)\end{array}$} & \multicolumn{1}{c}{$\begin{array}{c}r_{\lim } \\
\left({ }^{\prime}\right)\end{array}$} & $\begin{array}{c}\mu_{\mathrm{V}, \lim } \\
\left(\mathrm{mag} \mathrm{arcsec}^{-2}\right)\end{array}$ & \multicolumn{1}{c}{} \\
\hline Pal 13 & 26.0 & 26.9 & -42.70 & 11.2 & 30.96 & -7.93 \\
NGC 7492 & 26.3 & 25.3 & -63.48 & 9.6 & 29.81 & 10.78 \\
Whiting 1 & 30.1 & 34.5 & -60.76 & 5.8 & 30.29 & 17.41 \\
NGC 6229 & 30.5 & 29.8 & 40.31 & 13.4 & 30.52 & -7.20 \\
AM 4 & 32.2 & 27.8 & 33.51 & 5.9 & 30.06 & -4.92 \\
Ko 2 & 34.7 & 41.9 & 25.54 & 4.8 & 31.27 & -11.50 \\
NGC 5694 & 35.0 & 29.4 & 30.36 & 12.3 & 30.24 & -9.02 \\
NGC 7006 & 41.2 & 38.5 & -19.41 & 14.9 & 29.89 & 0.75 \\
Pal 14 & 76.5 & 71.6 & 42.19 & 9.6 & 30.69 & \\
NGC 2419 & 82.6 & 89.9 & 25.24 & 8.8 & 29.49 & \\
Eridanus & 90.1 & 95.0 & -41.33 & 10.5 & 30.84 & \\
Pal 3 & 92.5 & 95.7 & 41.86 & 7.2 & 30.74 & \\
Pal 4 & 108.7 & 111.2 & 71.80 & 6.7 & 31.25 & \\
AM 1 & 123.3 & 124.6 & -48.47 & 3.6 & 31.63 & \\
\hline
\end{tabular}

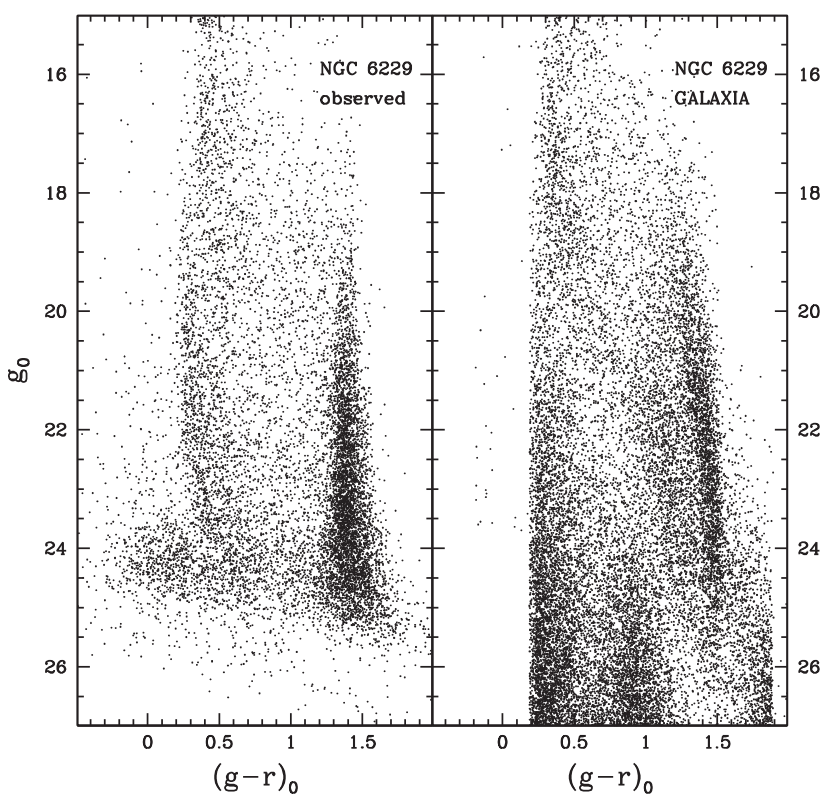

Figure 3. Comparison between the observed CMD of NGC 6229 (left-hand panel) and the synthetic CMD predicted by the GALAXIA model (right-hand panel).

The number $N_{\text {pop }}$ of synthetic stars within $3 \sigma$ about the cluster MS ridge line and in the absolute magnitude range $3.92<M_{\mathrm{g}}<6$ was similarly computed. The corresponding surface brightness was then estimated as:

$\mu_{\mathrm{V}, \text { syn }}=M_{\mathrm{V}, \text { tot }}+\mathrm{DM}_{\mathrm{GC}}-2.5 \log \left(\frac{N_{\mathrm{syn}}}{N_{\mathrm{pop}}}\right)+17.78$.

We do not include the observational effects (photometric errors, incompleteness, and contamination from background galaxies). These effects are indeed hard to be properly quantified in our data and they are not expected to be significant in the magnitude range where our analysis is conducted $(g<24)$.

This procedure was repeated $10^{4}$ times and the corresponding distributions of $N_{\text {syn }}$ and $\mu_{\mathrm{V} \text {, syn }}$ were computed. The number of stars contained within the same magnitude limits defined above was counted in the observed CMD and the density of stars per square degree $\Sigma_{\text {obs }}$ was calculated by dividing by the sampled area.
The fractions $f_{N}$ of random extractions with $N_{\text {syn }}<\Sigma_{\text {obs }}$ and the fractions $f_{\mu}$ with $\mu_{\mathrm{V} \text {, mod }}>\mu_{\mathrm{V} \text {, max }}$ were then computed.

If the MS star density estimated around the sample of observed GCs were compatible with that predicted by models, the distribution of percentiles would be uniform. In Fig. 7, the cumulative distributions of percentiles of the eight GCs at $d_{\odot}<45 \mathrm{kpc}$ with respect to the $N_{\text {syn }}$ distribution of the five Aquarius simulations are shown. On average, the distribution of percentiles is consistent with a uniform distribution (although a vast range of lumpiness is covered by the five considered simulations), indicating a general agreement with model predictions for three out of five simulations. A KolmogorovSmirnov (KS) test indicates probabilities $0.1<P<0.2$ for these simulations. However, the simulations Aquarius D and Aquarius E show opposite trends with distributions of percentiles that are incompatible with observations. Note that Aquarius D is the most massive halo among the five simulations of the Aquarius sample, with a virial mass $M=2.52 \times 10^{12} \mathrm{M}_{\odot}$, exceeding the current estimate for the Milky Way (Patel, Besla \& Mandel 2017). It is therefore likely that the number density of the halo is also overestimated in this simulation. On the other hand, simulation Aquarius E is the one with the lowest fraction of stars in the diffuse halo at distances $d_{G C}>20 \mathrm{kpc}$, with the majority of stars in this distance range contained in bound satellites. For this reason, the number density outside satellites turns out to be lower than those in other haloes.

The same conclusion can be drawn when the entire sample of $14 \mathrm{GCs}$ is considered in the $\mu_{V}$ space. The distributions of extracted surface brightness from each of the considered simulations are shown as a function of heliocentric distance in Fig. 8. We note that the background surface brightness distribution is peaked at values in the range $30<\mu_{\mathrm{V} \text {, syn }}<35 \mathrm{mag} \operatorname{arcsec}^{-2}$ following a declining trend with distance. At large distances, the surface brightness distribution becomes skewed with a tail towards bright values at $\mu_{\mathrm{V} \text {, syn }}<34 \mathrm{mag} \operatorname{arcsec}^{-2}$. This is a consequence of the increasing degree of lumpiness of the halo at large distances: GCs forming within satellites in an early stage of dissolution are still surrounded by the compact remnant of their host galaxy. The upper limits provided in Section 3 all lie at surface brightness levels that are brighter than the synthetic distributions within all simulations, the only exception being simulation Aquarius $\mathrm{D}$, which predicts a high surface brightness incompatible with the data of GCs at $d_{\odot}<50 \mathrm{kpc}$ (see above). Since our estimates are only upper limits to the actual surface brightness levels, the probability they are compatible with the distributions predicted by a given model is

$P=\prod_{i=1}^{14} f_{\mu}$

The derived probabilities are in the range $0.11<P<0.75$, compatible with no significant difference from the data, again with the exception of simulation Aquarius $\mathrm{D}$, which has $P<10^{-5}$.

An alternative approach is to apply the same analysis performed on data to the mock catalogues generated from the Aquarius simulations. Note that, among the three different techniques described in Section 3, only methods A and C can be applied, while method B (based on the ratio of number counts in regions populated mainly by halo or disc stars) cannot be used since the Aquarius simulations do not include a disc population. The detection probability $\left(P_{i}\right)$ of each of the eight GCs at $d_{\odot}<45 \mathrm{kpc}$ was estimated by applying these two techniques to each of the $10^{4}$ Monte Carlo synthetic CMDs constructed from each of the five Aquarius simulations. Then, a set of eight random numbers $\left(\eta_{i}\right)$ uniformly distributed between 0 and 1 was extracted and associated with the 'synthetic GCs'. The number 

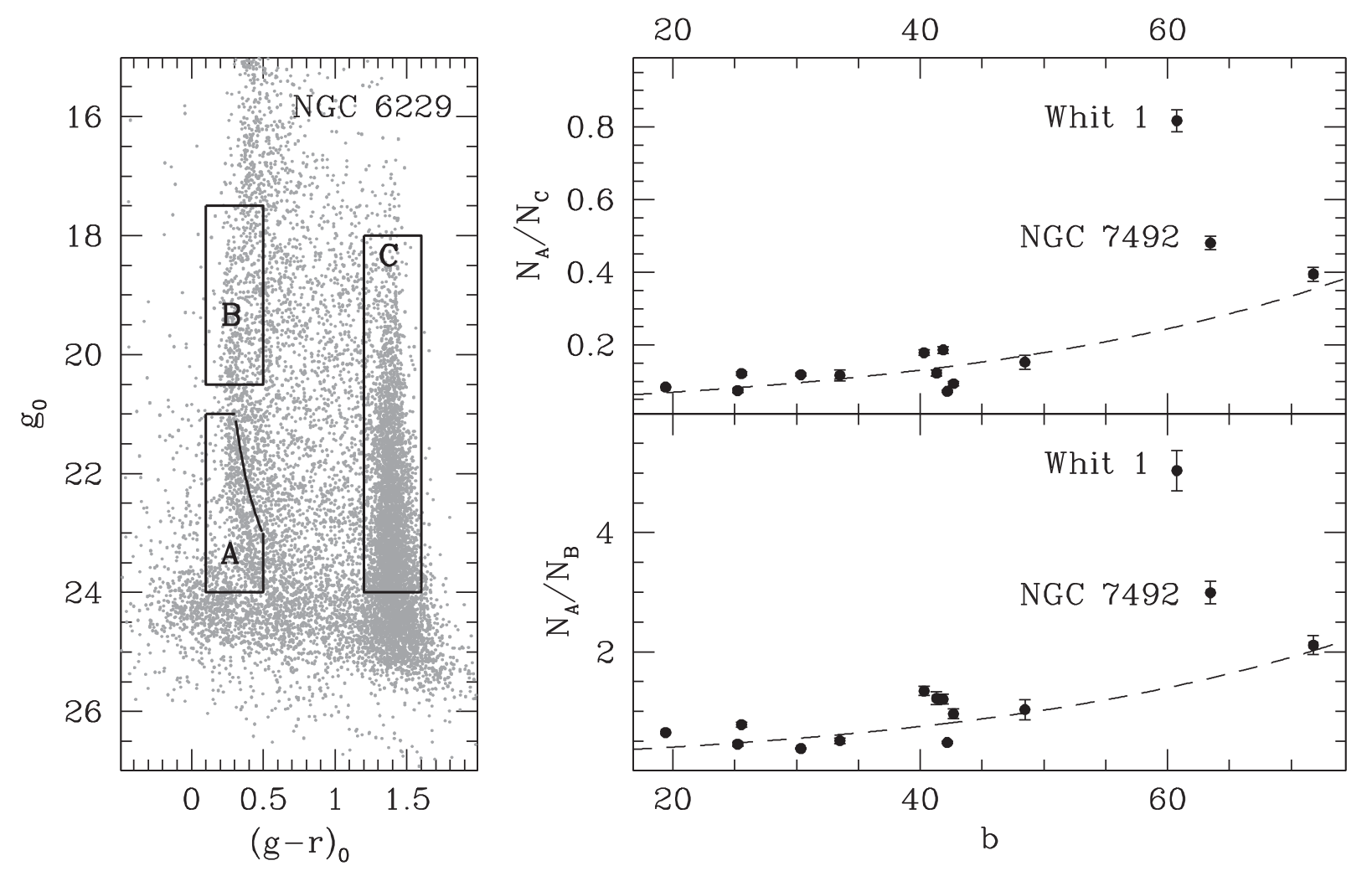

Figure 4. Left-hand panel: CMD of NGC 6229. The adopted selection boxes are indicated. Right-hand panels: number count ratios as a function of Galactic latitude. The Galactic trend predicted by the GALAXIA model is indicated in both panels with dashed lines.

of detected 'synthetic GCs' was calculated as the number of times the inequality $\eta_{i}<P_{i}$ is verified. The above procedure was repeated $10^{4}$ times and the distribution of detections was constructed (see Fig. 9). It can be seen that while method A tends to be more efficient than method $\mathrm{C}$ in detecting underlying remnants (at the cost of being dependent on the adequacy of the GALAXIA model), in both cases the probability of detecting $\leq 2 \mathrm{GCs}$ is non-negligible.

It is interesting to investigate the detection efficiency of our analysis as a function of the characteristics of the progenitor host galaxies of GCs. To do this, for each of the $10^{4}$ sets of 'synthetic GCs' extracted from a given Aquarius simulation (see above), we selected those particles located within 1 square degree around the 'synthetic GCs' and calculated the median redshift and absolute magnitude of their former host galaxies at the moment of their infall within the Milky Way halo. In this case, we considered the entire sample of 14 GCs and assumed as 'detected' any remnant producing a surface

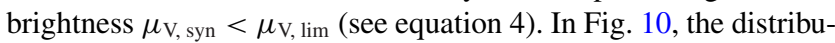
tions of detections in this diagram are shown for the five Aquarius simulations. Note that detected remnants occupy only a portion of this diagram at $z_{\text {infall }}<6$ and $M_{\mathrm{g}}<-10$, with small variations among the considered simulations. So, while it is not surprising that we were able to detect the Sagittarius stream (at $z_{\text {infall }} \sim 1$, $M_{\mathrm{g}, 0} \sim-13.8$; Law \& Majewski 2010b), no further bright satellite accretion seems to have contributed to the Galactic GC system in recent epochs in this Galactocentric distance range.

\section{DISCUSSION}

By analysing the projected density of stars around 14 GCs populating the outer halo of the Milky Way (at Galactocentric distances $d_{\mathrm{GC}}>25 \mathrm{kpc}$ ), we find evidence of significant overdensities surrounding two clusters, NGC 7492 and Whiting 1. No similar features were detected in any of the other surveyed GCs.

The presence of such extended, compact, and conspicuous stellar populations around these two GCs is confirmed through three independent techniques and cannot be associated with either the clusters themselves (because of their homogeneous spatial distribution across the field of view) or with the Galactic field (because of their CMD morphology). They are therefore compatible with the existence of a galactic remnant surrounding these GCs. The presence of overdensities around these two GCs was suggested by Carballo-Bello et al. (2014) who classified the detection around Whiting 1 as 'probable' and that around NGC 7492 as 'uncertain'.

The $N$-body simulation of Peñarrubia et al. (2010) predicts the presence of the Sagittarius stream at a coincident distance in the same portion of the sky occupied by Whiting 1 and NGC 7492, although only the former has a compatible radial velocity. In a recent spectroscopic follow-up, Carballo-Bello et al. (2017, 2018b) confirmed this hypothesis by revealing the presence of peaks in the velocity distribution of their sample that cannot be associated with the Galactic field. From these studies, they identified the presence of the Sagittarius stream in the neighbourhood of these GCs although only Whiting 1 seems to be kinematically linked to this substructure.

We do not confirm the detection of a conspicuous stream around NGC 2419 claimed by Newberg et al. (2003) (see also Ruhland et al. 2011) using star counts of blue horizontal branch stars and later identified as an RR Lyrae overdensity by Drake et al. (2013). Although at the distance of this remote GC, it is difficult to sample the CMD at the turn-off level with a good level of completeness, the large number of blue horizontal branch stars and RR Lyrae would imply a surface brightness well above the limit reached by our data. 

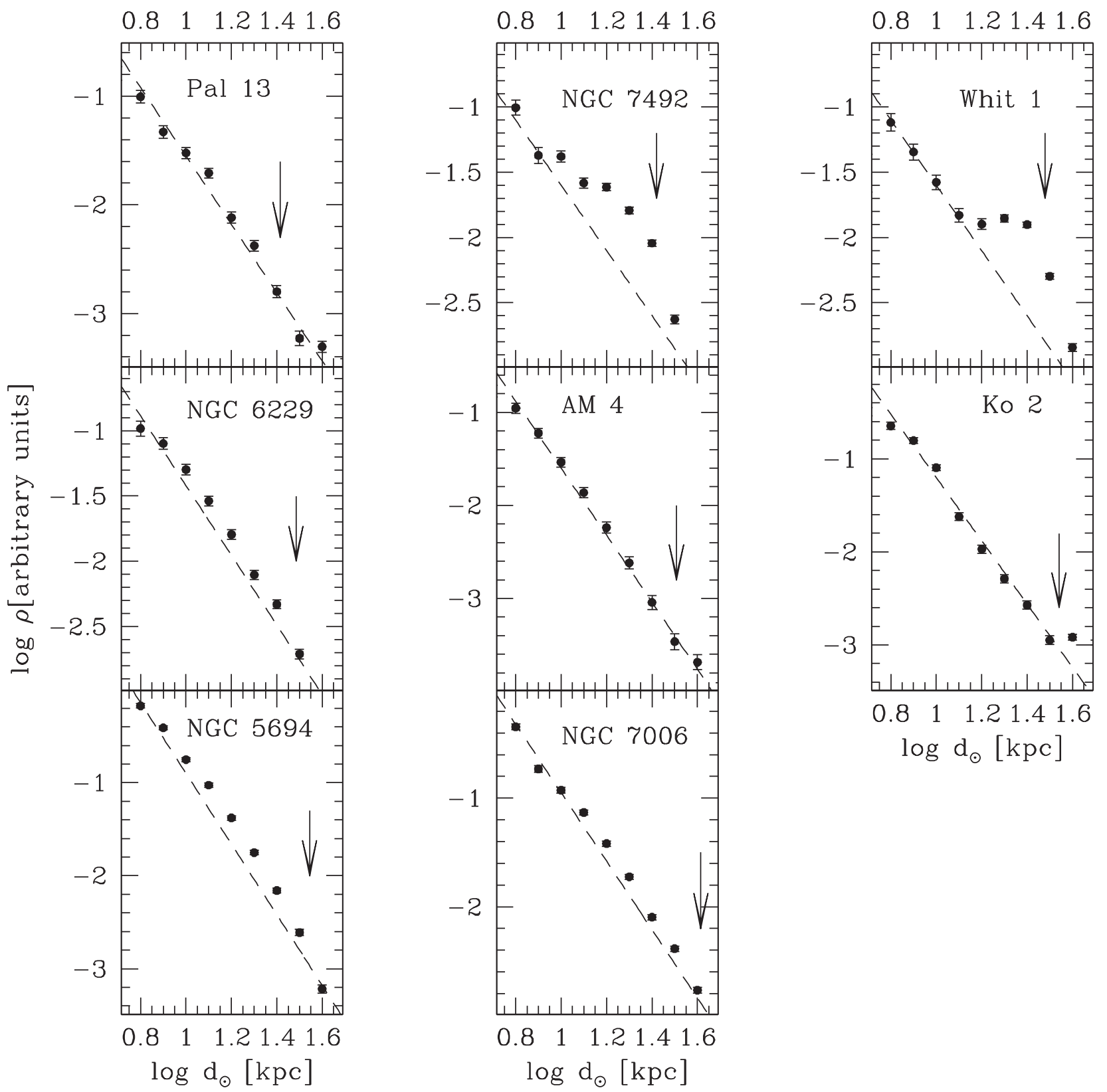

Figure 5. 3D density profiles of the eight GCs at heliocentric distances $d_{\odot}<45 \mathrm{kpc}$. The power-law best fits are marked with dashed lines. The heliocentric distance of each cluster is indicated by arrows.

On the other hand, at distances $d_{\odot}>100 \mathrm{kpc}$, the turn-off region of any hypothetical stellar population would overlap with the locus of background galaxies, thus hampering any significant detection. So, whether the observed overdensity is linked to the Virgo stellar stream (as hypothesised by Casetti-Dinescu et al. 2009) or to the outer portion of the trailing arm of the Sagittarius stream (Belokurov et al. 2014), they should lie beyond this distance.

The lack of detected streams around all the other GCs is, however, not surprising. Indeed, the comparison with five different Milky Way-like galaxies in the Aquarius cosmological simulation indicates that both the predicted mean stellar densities and the surface brightness of the halo at the distances of the surveyed GCs as well as the predicted detection efficiencies of the techniques employed in our analysis are still compatible with those measured in our survey, in agreement with what was found by Bell et al.
(2008); Starkenburg et al. (2009); Xue et al. (2011), and Janesh et al. (2016) . The limiting surface brightness of our survey is estimated to be $29.4<\mu_{\mathrm{V}, \max }<31.7 \mathrm{mag} \operatorname{arcsec}^{-2}$ depending on the cluster distance and spatial coverage, and is about 2 mag brighter than those of typical satellites predicted by models in this range of distances. Moreover, a further complication arises at distances $>40 \mathrm{kpc}$ because of the noise due to the strong contamination from blue background galaxies that overlap with the turn-off region of any subjacent stellar population. Any possible discrepancy cannot be revealed by our survey and its detection would require deeper data collected over larger fields of view (provided that an efficient star/galaxy discrimination is available at very faint magnitudes).

Remarkably, in M31 the PAndAS survey revealed a wealth of compact substructures at distances $d>30 \mathrm{kpc}$ from its centre, with almost all GCs beyond $40 \mathrm{kpc}$ being associated with some of these 

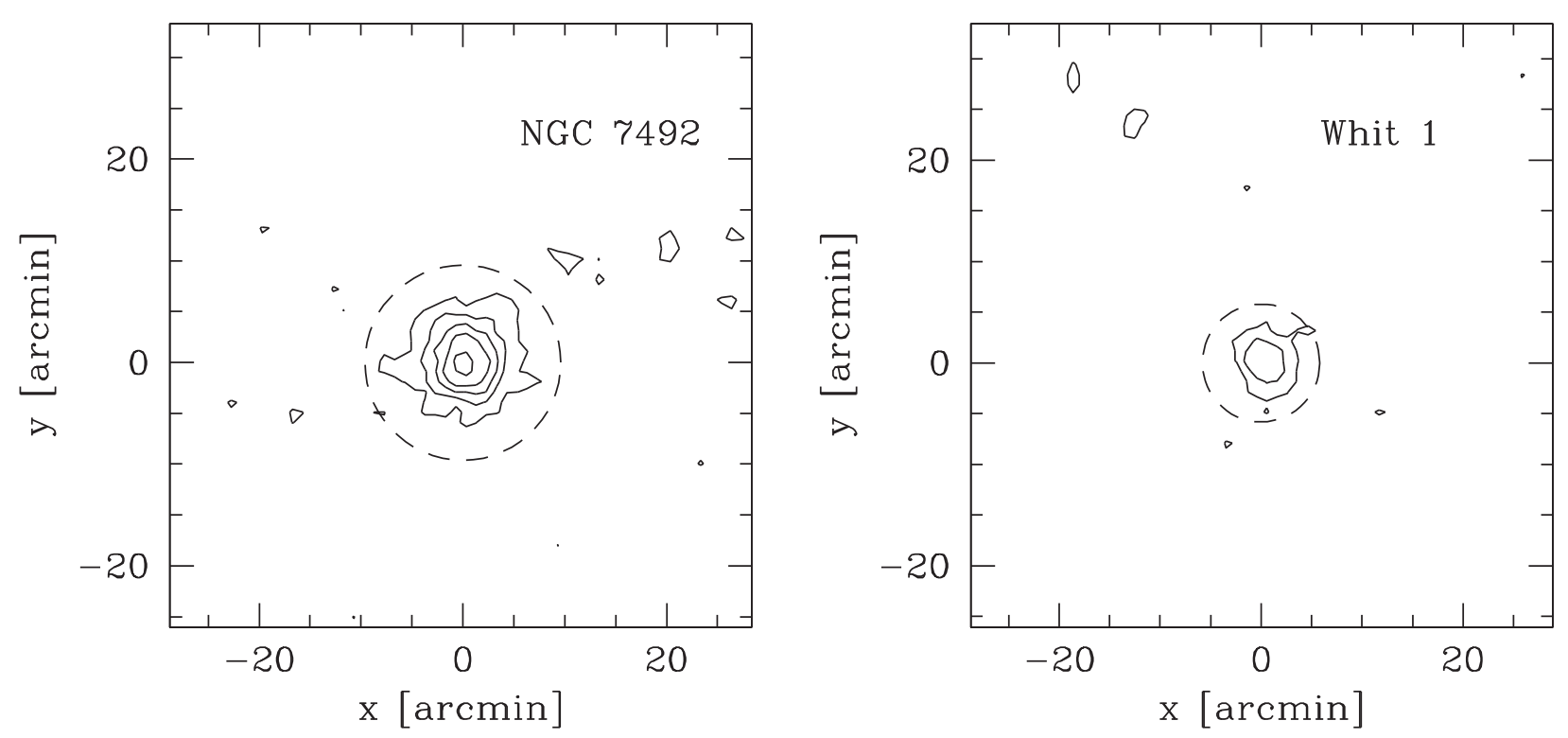

Figure 6. 2D density maps of NGC 7492 (left-hand panel) and Whiting 1 (right-hand panel). Density contours at $3 \sigma$ above the background are shown in logarithmic steps of 0.1 . The adopted limiting radii $r_{\mathrm{lim}}$ are marked with dashed lines.

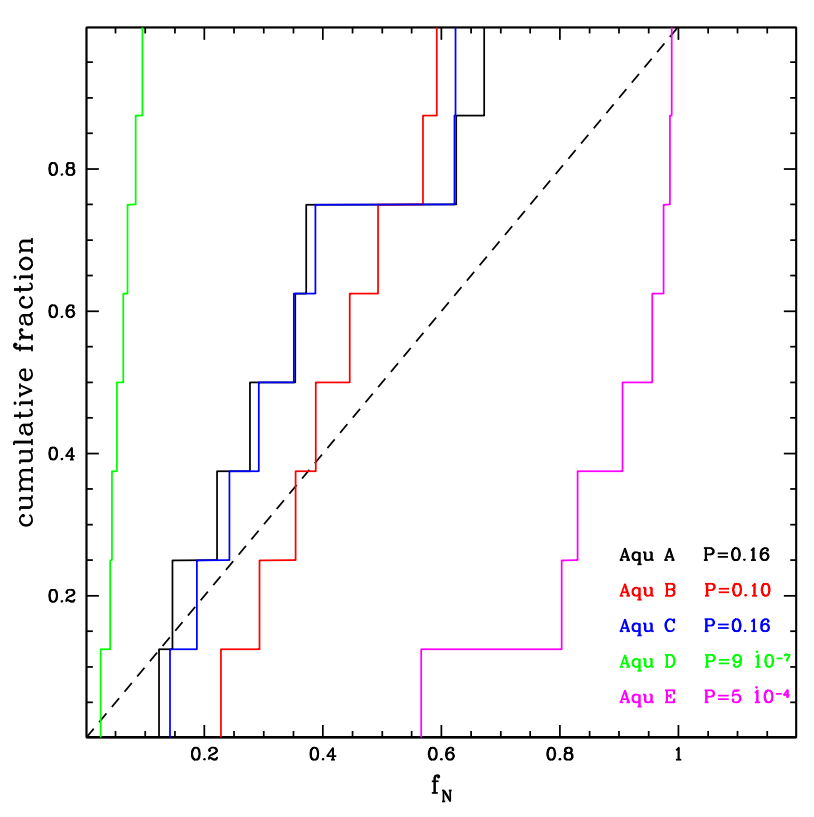

Figure 7. Cumulative distribution of percentiles in the number density domain for the eight GCs at $d_{\odot}<45 \mathrm{kpc}$. Different lines refer to the various cosmological simulations. The probabilities associated with the KS test are also indicated.

features (McConnachie et al. 2009; Mackey et al. 2010). A different picture would appear if an external view of the Milky Way could be obtained in a survey of similar depth, with only a few GCs aligned with the path of the Sagittarius stream. This indicates that, in spite of their similar masses and morphologies, M31 experienced a turbulent history characterized by many more accretion events than in the Milky Way.

The simulations used here for comparison include several approximations that could make them less than ideal in this context. For instance, they do not include the contribution and the dynamical effect of the halo component formed in situ from the gas accreted in past mergers or the baryonic disc(s). Besides these intrinsic limitations of the simulations, the analysis performed in Section 4 contains many approximations and uncertain assumptions (number density-surface brightness conversion, photometric completeness, stellar population synthesis, etc.) and neglects the variation of the GC formation/destruction efficiency in different host galaxies, thus making a proper quantitative comparison between models and observations uncertain.

Further constraints based on wide-field surveys of the halo stellar content at faint magnitudes, like those provided by the future Large Synoptic Survey Telescope (Ivezic et al. 2008), are clearly needed for a sound estimate of the degree of lumpiness of the halo at distances beyond $40 \mathrm{kpc}$.

\section{ACKNOWLEDGEMENTS}

DMD and EKG gratefully acknowledge support by the Sonderforshungsbereich 'The Milky Way System' (SFB 881, especially through subprojects A2 and A8) of the German Research Foundation (DFG). RRM acknowledges partial support from the BASAL PFB-06 project as well as FONDECYT project $\mathrm{N}^{\circ} 1170364$. JAC-B acknowledges financial support received from the CONICYT-Chile FONDECYT Postdoctoral Fellowship 3160502, from the Ministry for the Economy, Development, and Tourism's Programa Iniciativa Científica Milenio through grant IC120009, awarded to the Millennium Institute of Astrophysics (MAS), and from CONICYT's PCI program through grant DPI20140066. SGD acknowledges a partial support from the NSF grants AST-1313422, AST-1413600, and AST-1518308. This work is based on observations obtained with MegaCam, a joint project of CFHT and CEA/DAPNIA, at the CFHT observing programs 09AF03 (PI: Valls-Gabaud), 09AC07, 09BC02, and 10AC06 (PI: Côté), on data products produced at TERAPIX and the Canadian Astronomy Data Centre, and on data gathered with the 6.5 metre Magellan Telescopes located at Las Campanas Observatory, Chile under the observing program 2010B0472 (PI: Geha). We warmly thank Michele Bellazzini for useful discussions. 


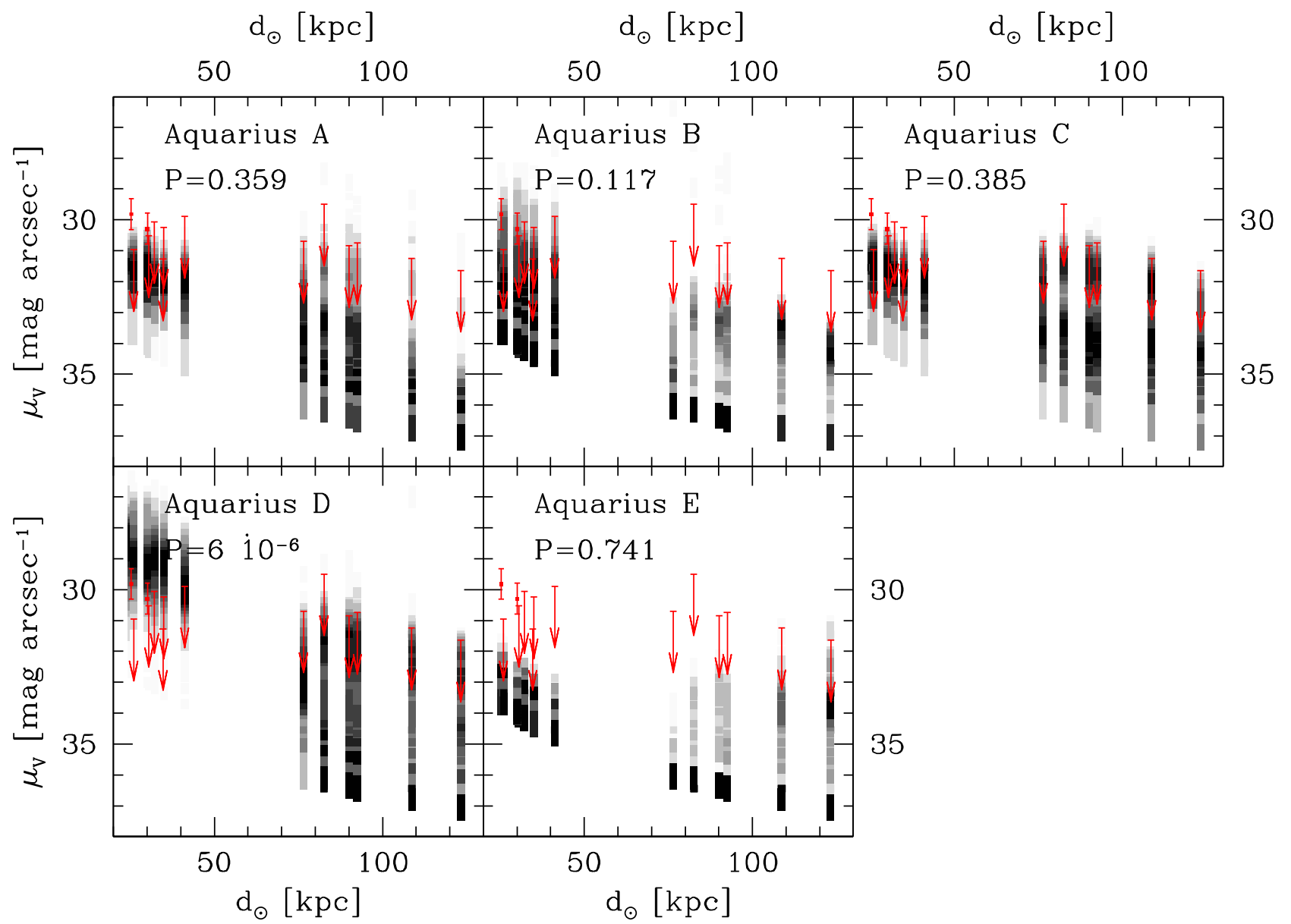

Figure 8. Distribution of extracted surface brightness as a function of the heliocentric distance. Darker regions indicate a higher number of extractions. The upper limits for the sample GCs are indicated by arrows.

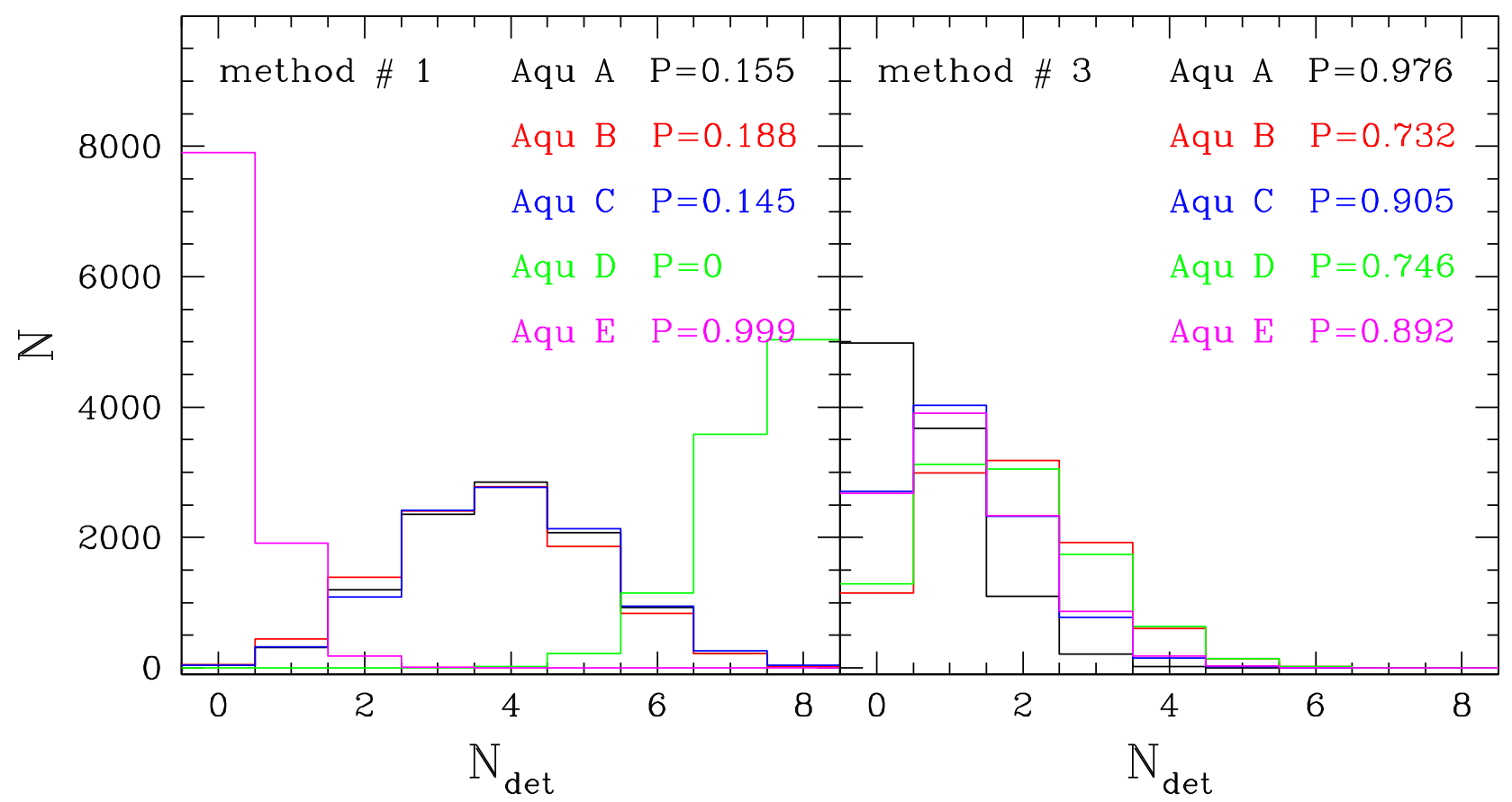

Figure 9. Histograms of detections in the sample of mock catalogues generated from the five Aquarius simulations. The left-hand panel refers to method A while the right-hand panel refers to method C. Different lines refer to the various cosmological simulations. The probabilities of detecting $\leq 2$ underlying remnants are also indicated. 


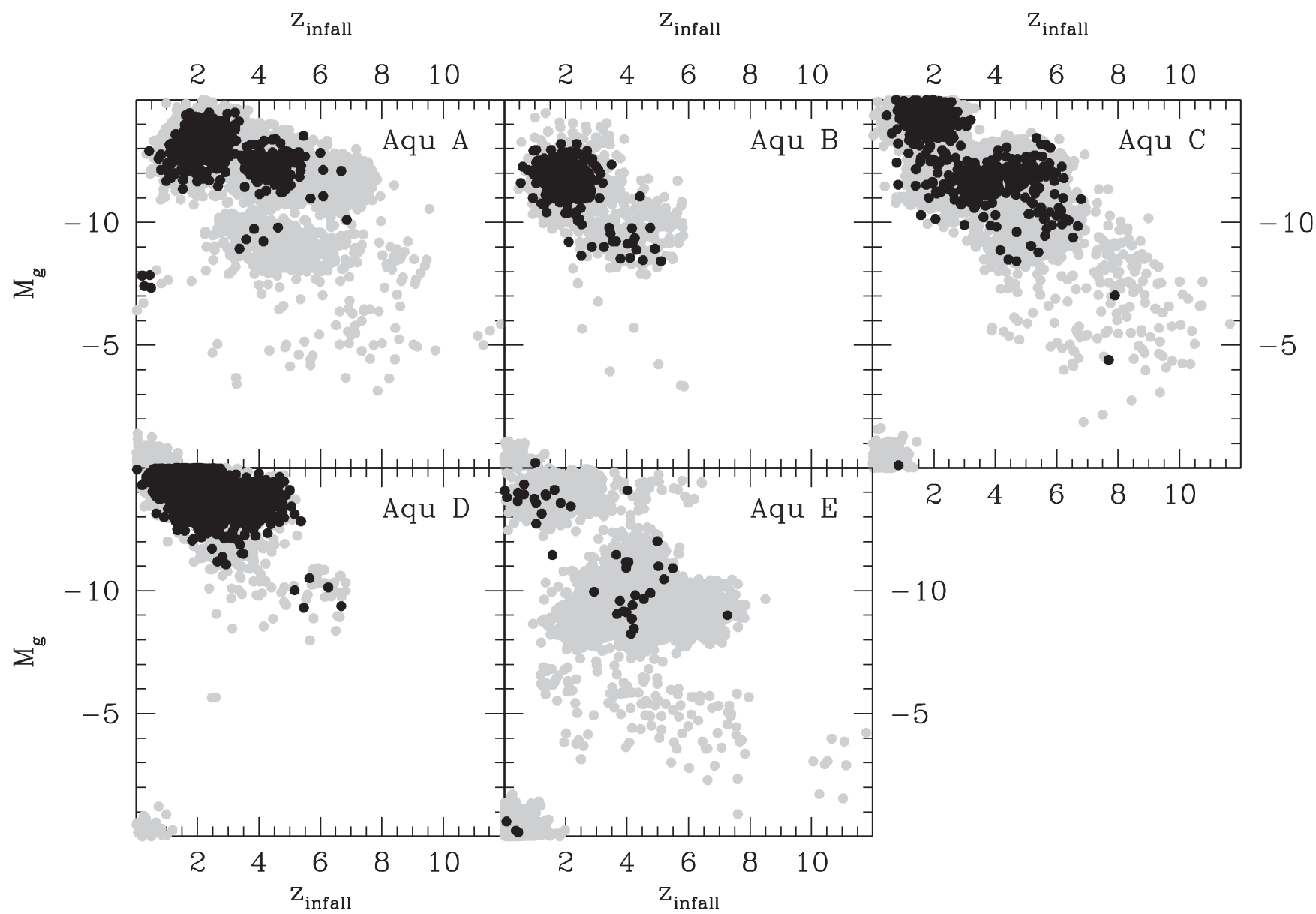

Figure 10. Distributions of detections in the sample of mock catalogues generated from the five Aquarius simulations in the infall redshift versus host absolute $g$ magnitude. Grey dots mark the location in this plane of all extractions, black dots mark the location of detections.

\section{REFERENCES}

Abazajian K. N. et al., 2009, ApJS, 182, 543

Bell E. F. et al., 2008, ApJ, 680, 295

Bellazzini M., Ibata R., Ferraro F. R., Testa V., 2003, A\&A, 405, 577

Belokurov V. et al., 2006, ApJ, 642, L137

Belokurov V. et al., 2014, MNRAS, 437, 116

Boulade O. et al., 2003, Proc. SPIE, 4841, 72

Bradford J. D. et al., 2011, ApJ, 743, 167

Bressan A., Marigo P., Girardi L., Salasnich B., Dal Cero C., Rubele S., Nanni A., 2012, MNRAS, 427, 127

Bullock J. S., Johnston K. V., 2005, ApJ, 635, 931

Carballo-Bello J. A., Sollima A., Martínez-Delgado D., Pila-Díez B., Leaman R., Fliri J., Muñoz R. R., Corral-Santana J. M., 2014, MNRAS, 445, 2971

Carballo-Bello J. A., Muñoz R. R., Carlin J. L., Côté P., Geha M., Simon J. D., Djorgovski S. G., 2015, ApJ, 805, 51

Carballo-Bello J. A. et al., 2017, MNRAS, 467, L91

Carballo-Bello J. A., Martínez-Delgado D., Navarrete C., Catelan M, Muñoz R. R., Antoja T, Sollima A, 2018a, MNRAS, 474, 683

Carballo-Bello J. A. et al., 2018b, MNRAS, 474, 4766

Casetti-Dinescu D. I., Girard T. M., Majewski S. R., Vivas A. K., Wilhelm R., Carlin J. L., Beers T. C., van Altena W. F., 2009, ApJ, 701, L29

Cerviño M., Valls-Gabaud D., Luridiana V., Mas-Hesse J. M., 2002, A\&A, 381,51

Chambers K. C. et al., 2016, preprint (arXiv:1612.05560)

Cole S., Lacey C. G., Baugh C. M., Frenk C. S., 2000, MNRAS, 319, 168

Colless M. et al., 2001, MNRAS, 328, 1039

Cooper A. P. et al., 2010, MNRAS, 406, 744
Côté P., Marzke R. O., West M. J., 1998, ApJ, 501, 554

Côté P., Marzke R. O., West M. J., Minniti D., 2000, ApJ, 533, 869

Deason A. J., Belokurov V., Weisz D. R., 2015, MNRAS, 448, L77

Drake A. J. et al., 2013, ApJ, 765, 154

Duc P.-A. et al., 2015, MNRAS, 446, 120

Forbes D. A., Bridges T., 2010, MNRAS, 404, 1203

Gao L., Navarro J. F., Cole S., Frenk C. S., White S. D. M., Springel V., Jenkins A., Neto A. F., 2008, MNRAS, 387, 536

Griffen B. F., Drinkwater M. J., Thomas P. A., Helly J. C., Pimbblet K. A., 2010, MNRAS, 405, 375

Harris W. E., 1996, AJ, 112, 1487

Ibata R. A., Gilmore G., Irwin M. J., 1994, Nature, 370, 194

Ibata R., Irwin M., Lewis G., Ferguson A. M. N., Tanvir N., 2001, Nature, 412,49

Ibata R., Sollima A., Nipoti C., Bellazzini M., Chapman S. C., Dalessandro E., 2011, ApJ, 738, 186

Ivezic Z. et al., 2008, Serb. Astron. J., 176, 1

Janesh W. et al., 2016, ApJ, 816, 80

Keller S. C., Mackey D., Da Costa G. S., 2012, ApJ, 744, 57

King I. R., 1966, AJ, 71, 64

Kravtsov A. V., Gnedin O. Y., 2005, ApJ, 623, 650

Kuzma P. B., Da Costa G. S., Mackey A. D., 2018, MNRAS, 473, 2881

Law D. R., Majewski S. R., 2010a, ApJ, 718, 1128

Law D. R., Majewski S. R., 2010b, ApJ, 714, 229

Law D. R., Johnston K. V., Majewski S. R., 2005, ApJ, 619, 807

Leaman R., VandenBerg D. A., Mendel J. T., 2013, MNRAS, 436, 122

Lowing B., Wang W., Cooper A., Kennedy R., Helly J., Cole S., Frenk C., 2015, MNRAS, 446, 2274

Mackey A. D., Gilmore G. F., 2004, MNRAS, 355, 504 
Mackey A. D. et al., 2010, ApJ, 717, L11

Majewski S. R., Skrutskie M. F., Weinberg M. D., Ostheimer J. C., 2003, ApJ, 599, 1082

Marigo P. et al., 2017, ApJ, 835, 77

Marín-Franch A. et al., 2009, ApJ, 694, 1498

Martínez-Delgado D., Zinn R., Carrera R., Gallart C., 2002, ApJ, 573, L19

Martínez-Delgado D. et al., 2010, AJ, 140, 962

McConnachie A. W. et al., 2009, Nature, 461, 66

Muñoz R. R. et al., 2018, ApJ, submitted

Myeong G. C., Jerjen H., Mackey D., Da Costa G. S., 2017, ApJ, 840, L25

Newberg H. J. et al., 2003, ApJ, 596, L191

Pasetto S., Chiosi C., Kawata D., 2012, A\&A, 545, A14

Patel E., Besla G., Mandel K., 2017, MNRAS, 468, 3428

Pawlowski M. S., Pflamm-Altenburg J., Kroupa P., 2012, MNRAS, 423, 1109

Peñarrubia J., Belokurov V., Evans N. W., Martínez-Delgado D., Gilmore G., Irwin M., Niederste-Ostholt M., Zucker D. B., 2010, MNRAS, 408, L26

Pillepich A., Madau P., Mayer L., 2015, ApJ, 799, 184

Planck Collaboration et al., 2016, A\&A, 594, A1

Renaud F., Agertz O., Gieles M., 2017, MNRAS, 465, 3622

Rodriguez-Gomez V. et al., 2016, MNRAS, 458, 2371

Ruhland C., Bell E. F., Rix H.-W., Xue X.-X., 2011, ApJ, 731, 119
Santana F. A., Muñoz R. R., Geha M., Côté P., Stetson P., Simon J. D., Djorgovski S. G., 2013, ApJ, 774, 106

Schlafly E. F., Finkbeiner D. P., 2011, ApJ, 737, 103

Schlegel D. J., Finkbeiner D. P., Davis M., 1998, ApJ, 500, 525

Searle L., Zinn R., 1978, ApJ, 225, 357

Sharma S., Bland-Hawthorn J., Johnston K. V., Binney J., 2011, ApJ, 730, 3

Silverman B. W., 1986, Density Estimation for Statistics and Data Analysis. Chapman and Hall, London

Skrutskie M. F. et al., 2006, AJ, 131, 1163

Sollima A., Valls-Gabaud D., Martínez-Delgado D., Fliri J., Peñarrubia J., Hoekstra H., 2011a, ApJ, 730, L6

Sollima A., Martínez-Delgado D., Valls-Gabaud D., Peñarrubia J., 2011b, ApJ, 726, 47

Spergel D. N. et al., 2003, ApJS, 148, 175

Springel V. et al., 2008, MNRAS, 391, 1685

Starkenburg E. et al., 2009, ApJ, 698, 567

Stetson P. B., 1994, PASP, 106, 250

Trager S. C., King I. R., Djorgovski S., 1995, AJ, 109, 218

Veljanoski J. et al., 2014, MNRAS, 442, 2929

Xue X.-X. et al., 2011, ApJ, 738, 79

This paper has been typeset from a $\mathrm{T}_{\mathrm{E}} \mathrm{X} / \mathrm{LA} \mathrm{T}_{\mathrm{E}} \mathrm{X}$ file prepared by the author. 